\title{
The Fluorescence Detector of the Pierre Auger Observatory
}

\section{The Pierre Auger Collaboration}

J. Abraham ${ }^{8}$, P. Abreu ${ }^{71}$, M. Aglietta ${ }^{54}$, C. Aguirre ${ }^{12}$, E.J. Ahn ${ }^{87}$, D. Allard ${ }^{31}$, I. Allekotte ${ }^{1}$, J. Allen $^{90}$, P. Allison ${ }^{92}$, J. Alvarez-Muñiz ${ }^{78}$, M. Ambrosio ${ }^{48}$, L. Anchordoqui ${ }^{104}$, S. Andringa ${ }^{71}$, A. Anzalone ${ }^{53}$, C. Aramo $^{48}$, E. Arganda ${ }^{75}$, S. Argirò ${ }^{51}$, K. Arisaka ${ }^{95}$, F. Arneodo ${ }^{55}$,

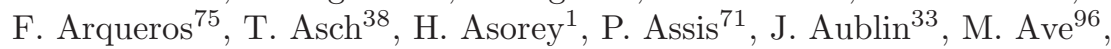
G. Avila ${ }^{10}$, A. Bacher ${ }^{38}$, T. Bäcker ${ }^{42}$, D. Badagnani ${ }^{6}$, K.B. Barber ${ }^{11}$, A.F. Barbosa ${ }^{14}$, H.J.M. Barbosa ${ }^{17}$, N. Barenthien ${ }^{41}$, S.L.C. Barroso ${ }^{20}$, B. Baughman ${ }^{92}$, P. Bauleo ${ }^{85}$, J.J. Beatty ${ }^{92}$, T. Beau ${ }^{31}$, B.R. Becker ${ }^{101}$, K.H. Becker ${ }^{36}$, A. Bellétoile ${ }^{34}$, J.A. Bellido ${ }^{11,93}$, S. BenZvi ${ }^{103}$, C. Berat ${ }^{34}$, P. Bernardini ${ }^{47}$, X. Bertou ${ }^{1}$, P.L. Biermann ${ }^{39}$, P. Billoir ${ }^{33}$, O. Blanch-Bigas ${ }^{33}$, F. Blanco ${ }^{75}$, C. Bleve ${ }^{47}$, H. Blümer ${ }^{41,37}$, M. Boháčováa ${ }^{96,27}$, E. Bollmann ${ }^{37}$, H. Bolz ${ }^{37}$, C. Bonifazi ${ }^{33}$, R. Bonino ${ }^{54}$, N. Borodai ${ }^{69}$, F. Bracci $^{49}$, J. Brack ${ }^{85}$,

P. Brogueira ${ }^{71}$, W.C. Brown ${ }^{86}$, R. Bruijn ${ }^{81}$, P. Buchholz ${ }^{42}$, A. Bueno ${ }^{77}$, R.E. Burton ${ }^{83}$, N.G. Busca ${ }^{31}$, K.S. Caballero-Mora ${ }^{41}$, D. Camin ${ }^{46}$

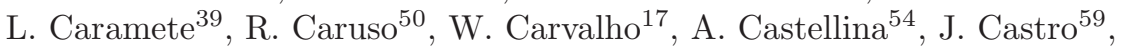
O. Catalano ${ }^{53}$, L. Cazon ${ }^{96}$, R. Cester ${ }^{51}$, J. Chauvin ${ }^{34}$, A. Chiavassa ${ }^{54}$, J.A. Chinellato ${ }^{18}$, A. $\mathrm{Chou}^{87}{ }^{90}$, J. Chudoba ${ }^{27}$, J. Chye ${ }^{89}$, P.D.J. Clark ${ }^{81}$, R.W. Clay $^{11}$, E. Colombo ${ }^{2}$, R. Conceição ${ }^{71}$, B. Connolly ${ }^{102}$, F. Contreras ${ }^{9}$, J. Coppens ${ }^{65,67}$, A. Cordero ${ }^{59}$, A. Cordier ${ }^{32}$, U. Cotti ${ }^{63}$, S. Coutu ${ }^{93}$, C.E. Covault ${ }^{83}$, A. Creusot ${ }^{73}$, A. Criss $^{93}$, J.W. Cronin ${ }^{96}$, J. Cuautle ${ }^{59}$, A. Curutiu ${ }^{39}$, S. Dagoret-Campagne ${ }^{32}$, R. Dallier ${ }^{35}$, F. Daudo ${ }^{51}$,

K. Daumiller ${ }^{37}$, B.R. Dawson ${ }^{11}$, R.M. de Almeida ${ }^{18}$, M. De Domenico ${ }^{50}$,

C. De Donato ${ }^{46}$, S.J. de Jong ${ }^{65}$, G. De La Vega ${ }^{8}$, W.J.M. de Mello Junior ${ }^{18}$, J.R.T. de Mello $\mathrm{Neto}^{23}$, I. De Mitri ${ }^{47}$, V. de Souza ${ }^{16}$, K.D. de Vries ${ }^{66}$, G. Decerprit ${ }^{31}$, L. del Peral ${ }^{76}$, O. Deligny ${ }^{30}$, A. Della Selva ${ }^{48}$, C. Delle Fratte $^{49}$, H. Dembinski ${ }^{40}$, C. Di Giulio ${ }^{49}$, J.C. Diaz ${ }^{89}$, P.N. Diep ${ }^{105}$, C. Dobrigkeit ${ }^{18}$, J.C. D'Olivo ${ }^{64}$, P.N. Dong ${ }^{105}$, D. Dornic ${ }^{30}$, A. Dorofeev ${ }^{88}$, J.C. dos Anjos ${ }^{14}$, M.T. Dova ${ }^{6}$, D. D’Urso ${ }^{48}$, I. Dutan ${ }^{39}$, M.A. DuVernois ${ }^{98}$, R. Engel ${ }^{37}$, M. Erdmann ${ }^{40}$, C.O. Escobar ${ }^{18}$, A. Etchegoyen ${ }^{2}$, P. Facal San Luis $^{96,78}$, H. Falcke ${ }^{65,68}$, G. Farrar ${ }^{90}$, A.C. Fauth ${ }^{18}$, N. Fazzini ${ }^{87}$, F. Ferrer ${ }^{83}$, A. Ferrero ${ }^{2}$, B. Fick ${ }^{89}$, A. Filevich ${ }^{2}$, A. Filipčič ${ }^{72,73}$, I. Fleck ${ }^{42}$, S. Fliescher ${ }^{40}$, R. Fonte ${ }^{50}$, C.E. Fracchiolla ${ }^{85}$, E.D. Fraenkel ${ }^{66}$, W. Fulgione ${ }^{54}$, R.F. Gamarra ${ }^{2}$, S. Gambetta ${ }^{44}$, B. García ${ }^{8}$, D. García Gámez ${ }^{77}$, D. Garcia-Pinto ${ }^{75}$, X. Garrido ${ }^{37,32}$, H. Geenen ${ }^{36}$, G. Gelmini ${ }^{95}$,

H. Gemmeke ${ }^{38}$, P.L. Ghia ${ }^{30,54}$, U. Giaccari ${ }^{47}$, K. Gibbs ${ }^{96}$, M. Giller ${ }^{70}$, J. Gitto ${ }^{7}$, H. Glass ${ }^{87}$, L.M. Goggin ${ }^{104}$, M.S. Gold ${ }^{101}$, G. Golup ${ }^{1}$, F. Gomez Albarracin $^{6}$, M. Gómez Berisso ${ }^{1}$, P.F. Gomez Vitale ${ }^{9}$ P. Gonçalves ${ }^{71}$, M. Gonçalves do Amaral ${ }^{24}$, D. Gonzalez ${ }^{41}$, J.G. Gonzalez ${ }^{77,88}$, D. Góra ${ }^{41,69}$, A. Gorgi ${ }^{54}$, P. Gouffon ${ }^{17}$, E. Grashorn ${ }^{92}$, V. Grassi ${ }^{46}$, S. Grebe ${ }^{65}$, M. Grigat ${ }^{40}$, A.F. Grillo ${ }^{55}$, J. Grygar ${ }^{27}$, Y. Guardincerri ${ }^{4}$, N. Guardone ${ }^{50}$, C. Guerard ${ }^{41}$, F. Guarino ${ }^{48}$, R. Gumbsheimer ${ }^{37}$, G.P. Guedes ${ }^{19}$, J. Gutiérrez ${ }^{76}$, 
J.D. Hague ${ }^{101}$, V. Halenka ${ }^{28}$, P. Hansen ${ }^{6}$, D. Harari ${ }^{1}$, S. Harmsma ${ }^{66,67}$, S. Hartmann ${ }^{36}$, J.L. Harton ${ }^{85}$, A. Haungs ${ }^{37}$, M.D. Healy ${ }^{95}$, T. Hebbeker ${ }^{40}$, G. Hebrero ${ }^{76}$, D. Heck ${ }^{37}$, C. Hojvat ${ }^{87}$, V.C. Holmes ${ }^{11}$, P. Homola ${ }^{69}$, G. Hofman ${ }^{86}$, J.R. Hörandel ${ }^{65}$, A. Horneffer ${ }^{65}$, M. Horvat ${ }^{73}$,

M. Hrabovský ${ }^{28,27}$, H. Hucker ${ }^{37}$, T. Huege ${ }^{37}$, M. Hussain ${ }^{73}$, M. Iarlori ${ }^{45}$, A. Insolia ${ }^{50}$, F. Ionita ${ }^{96}$, A. Italiano ${ }^{50}$, S. Jiraskova ${ }^{65}$, M. Kaducak ${ }^{87}$, K.H. Kampert ${ }^{36}$, T. Karova ${ }^{27}$, P. Kasper ${ }^{87}$, B. Kégl ${ }^{32}$, B. Keilhauer ${ }^{37}$, E. Kemp ${ }^{18}$, H. Kern ${ }^{37}$, R.M. Kieckhafer ${ }^{89}$, H.O. Klages ${ }^{37}$, M. Kleifges ${ }^{38}$, J. Kleinfeller ${ }^{37}$, R. Knapik ${ }^{85}$, J. Knapp ${ }^{81}$, D.-H. Koang ${ }^{34}$, A. Kopmann ${ }^{38}$,

A. Krieger ${ }^{2}$, O. Krömer ${ }^{38}$, D. Kruppke-Hansen ${ }^{36}$, D. Kuempel ${ }^{36}$, N. Kunka ${ }^{38}$,

A. Kusenko ${ }^{95}$, G. La Rosa ${ }^{53}$, C. Lachaud ${ }^{31}$, B.L. Lago ${ }^{23}$, P. Lautridou ${ }^{35}$,

M.S.A.B. Leão ${ }^{22}$, D. Lebrun ${ }^{34}$, P. Lebrun ${ }^{87}$, J. Lee ${ }^{95}$, M.A. Leigui de Oliveira $^{22}$, A. Lemiere ${ }^{30}$, A. Letessier-Selvon ${ }^{33}$, M. Leuthold ${ }^{40}$,

I. Lhenry-Yvon ${ }^{30}$, R. López ${ }^{59}$, A. Lopez Agüera ${ }^{78}$, K. Louedec ${ }^{32}$, J. Lozano Bahilo $^{77}$, A. Lucero ${ }^{54}$, H. Lyberis ${ }^{30}$, M.C. Maccarone ${ }^{53}$, C. Macolino ${ }^{45}$, S. Maldera ${ }^{54}$, M. Malek ${ }^{87}$, D. Mandat ${ }^{27}$, P. Mantsch $^{87}$, F. Marchetto ${ }^{51}$,

A.G. Mariazzi ${ }^{6}$, I.C. Maris ${ }^{41}$, H.R. Marquez Falcon ${ }^{63}$, D. Martello ${ }^{47}$,

O. Martineau ${ }^{37}$, O. Martínez Bravo ${ }^{59}$, H.J. Mathes ${ }^{37}$, J. Matthews ${ }^{88,94}$, J.A.J. Matthews ${ }^{101}$, G. Matthiae ${ }^{49}$, D. Maurizio ${ }^{51}$, P.O. Mazur ${ }^{87}$,

M. McEwen ${ }^{76}$, R.R. McNeil ${ }^{88}$, G. Medina-Tanco ${ }^{64}$, M. Melissas ${ }^{41}$, D. Melo ${ }^{51}$, E. Menichetti ${ }^{51}$, A. Menshikov ${ }^{38}$, R. Meyhandan ${ }^{88}$, M.I. Micheletti ${ }^{2}$, G. Miele ${ }^{48}$, W. Miller ${ }^{101}$, L. Miramonti ${ }^{46}$, S. Mollerach $^{1}$, M. Monasor ${ }^{75}$, D. Monnier Ragaigne ${ }^{32}$, F. Montanet ${ }^{34}$, B. Morales ${ }^{64}$, C. Morello ${ }^{54}$,

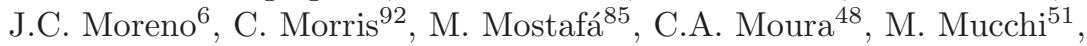
S. Mueller ${ }^{37}$, M.A. Muller ${ }^{18}$, R. Mussa ${ }^{51}$, G. Navarra ${ }^{54}$, J.L. Navarro ${ }^{77}$, S. Navas ${ }^{77}$, P. Necesal ${ }^{27}$, L. Nellen ${ }^{64}$, F. Nerling ${ }^{37}$, C. Newman-Holmes ${ }^{87}$, D. Newton ${ }^{81}$, P.T. Nhung ${ }^{105}$, D. Nicotra ${ }^{50}$, N. Nierstenhoefer ${ }^{36}$, D. Nitz ${ }^{89}$,

D. Nosek $^{26}$, L. Nožka ${ }^{27}$, M. Nyklicek ${ }^{27}$, J. Oehlschläger ${ }^{37}$, A. Olinto ${ }^{96}$, P. Oliva ${ }^{36}$, V.M. Olmos-Gilbaja ${ }^{78}$, M. Ortiz ${ }^{75}$, F. Ortolani ${ }^{49}$, B. Oßwald ${ }^{38}$, N. Pacheco ${ }^{76}$, D. Pakk Selmi-Dei ${ }^{18}$, M. Palatka ${ }^{27}$, J. Pallotta ${ }^{3}$, G. Parente ${ }^{78}$, E. Parizot $^{31}$, S. Parlati ${ }^{55}$, S. Pastor ${ }^{74}$, M. Patel ${ }^{81}$, T. Paul ${ }^{91}$, V. Pavlidou ${ }^{96} c^{\text {, }}$, K. Payet ${ }^{34}$, M. Pech ${ }^{27}$, J. Pȩkala ${ }^{69}$, I.M. Pepe ${ }^{21}$, L. Perrone ${ }^{56}$, R. Pesce ${ }^{44}$, E. Petermann ${ }^{100}$, S. Petrera ${ }^{45}$, P. Petrinca ${ }^{49}$, A. Petrolini ${ }^{44}$, Y. Petrov ${ }^{85}$, J. Petrovic ${ }^{67}$, C. Pfendner ${ }^{103}$, A. Pichel ${ }^{7}$, R. Piegaia ${ }^{4}$, T. Pierog ${ }^{37}$,

M. Pimenta ${ }^{71}$, T. Pinto ${ }^{74}$, V. Pirronello ${ }^{50}$, O. Pisanti ${ }^{48}$, M. Platino ${ }^{2}$, J. Pochon ${ }^{1}$, V.H. Ponce ${ }^{1}$, M. Pontz ${ }^{42}$, J. Pouryamout ${ }^{36}$, L. Prado Jr. ${ }^{18}$, P. Privitera ${ }^{96}$, M. Prouza ${ }^{27}$, E.J. Quel ${ }^{3}$, G. Raia ${ }^{57}$ J. Rautenberg ${ }^{36}$, O. Ravel ${ }^{35}$, D. Ravignani ${ }^{2}$, A. Redondo ${ }^{76}$, H.C. Reis ${ }^{18}$, S. Reucroft ${ }^{91}$, B. Revenu ${ }^{35}$, F.A.S. Rezende ${ }^{14}$, J. Ridky ${ }^{27}$, S. Riggi ${ }^{50}$, M. Risse $^{36}$,

C. Rivière ${ }^{34}$, V. Rizi ${ }^{45}$, C. Robledo ${ }^{59}$, M.D. Roberts ${ }^{93}$, G. Rodriguez ${ }^{49}$, J. Rodriguez Martino $^{50}$, J. Rodriguez Rojo ${ }^{9}$, I. Rodriguez-Cabo ${ }^{78}$,

M.D. Rodríguez-Frías ${ }^{76}$, G. $\operatorname{Ros}^{75,76}$, J. Rosado ${ }^{75}$, T. $\operatorname{Rossler}^{28}$, M. Roth $^{37}$, B. Rouillé-d'Orfeuil ${ }^{31}$, E. Roulet ${ }^{1}$, A.C. Rovero ${ }^{7}$, F. Salamida ${ }^{45}$,

H. Salazar ${ }^{59}$ b, G. Salina ${ }^{49}$, F. Sánchez ${ }^{64}$, M. Santander ${ }^{9}$, C.E. Santo ${ }^{71}$, E.M. Santos ${ }^{23}$, F. Sarazin ${ }^{84}$, S. Sarkar ${ }^{79}$, R. Sato ${ }^{9}$, N. Scharf ${ }^{40}$, V. Scherini ${ }^{36}$, H. Schieler ${ }^{37}$, P. Schiffer ${ }^{40}$, G. Schleif ${ }^{37}$ A. Schmidt ${ }^{38}$, F. Schmidt ${ }^{96}$, 
T. Schmidt ${ }^{41}$, O. Scholten ${ }^{66}$, H. Schoorlemmer ${ }^{65}$, J. Schovancova ${ }^{27}$, P. Schovánek ${ }^{27}$, F. Schroeder ${ }^{37}$, S. Schulte ${ }^{40}$, F. Schüssler ${ }^{37}$, D. Schuster ${ }^{84}$, S.J. Sciutto ${ }^{6}$, M. Scuderi ${ }^{50}$, A. Segreto ${ }^{53}$, D. Semikoz ${ }^{31}$, G. Sequieros ${ }^{51}$, M. Settimo ${ }^{47}$, R.C. Shellard ${ }^{14,15}$, I. Sidelnik ${ }^{2}$, B.B. Siffert ${ }^{23}$, A. Smiałkowski ${ }^{70}$, R. Šmída ${ }^{27}$, A.G.K. Smith ${ }^{11}$, B.E. Smith ${ }^{81}$, G.R. Snow ${ }^{100}$, P. Sommers ${ }^{93}$, J. Sorokin ${ }^{11}$, H. Spinka ${ }^{82,87}$, R. Squartini ${ }^{9}$, E. Strazzeri ${ }^{32}$, A. Stutz ${ }^{34}$, F. Suarez ${ }^{2}$, T. Suomijärvi ${ }^{30}$, A.D. Supanitsky ${ }^{64}$, M.S. Sutherland ${ }^{92}$, J. Swain ${ }^{91}$, Z. Szadkowski ${ }^{70}$, A. Tamashiro ${ }^{7}$, A. Tamburro ${ }^{41}$, T. Tarutina ${ }^{6}$, O. Taşcău ${ }^{36}$, R. Tcaciucc ${ }^{42}$, D. Tcherniakhovskii ${ }^{38}$, N.T. Thao ${ }^{105}$, D. Thomas ${ }^{85}$, R. Ticona ${ }^{13}$, J. Tiffenberg ${ }^{4}$, C. Timmermans ${ }^{67,65}$, W. Tkaczyk ${ }^{70}$, C.J. Todero

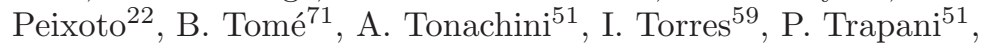

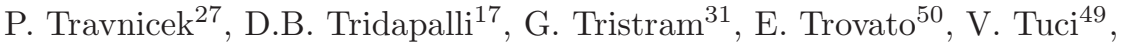
M. Tueros ${ }^{6}$, E. Tusi ${ }^{49}$, R. Ulrich ${ }^{37}$, M. Unger ${ }^{37}$, M. Urban ${ }^{32}$, J.F. Valdés Galicia $^{64}$, I. Valiño ${ }^{37}$, L. Valore ${ }^{48}$, A.M. van den Berg ${ }^{66}$, J.R. Vázquez ${ }^{75}$, R.A. Vázquez ${ }^{78}$, D. Veberič ${ }^{73,72}$, A. Velarde ${ }^{13}$, T. Venters ${ }^{96}$, V. Verzi ${ }^{49}$,

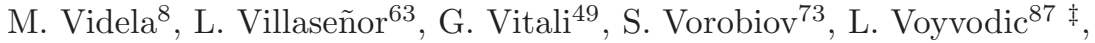
H. Wahlberg ${ }^{6}$, P. Wahrlich ${ }^{11}$, O. Wainberg ${ }^{2}$, D. Warner ${ }^{85}$, S. Westerhoff ${ }^{103}$, B.J. Whelan ${ }^{11}$, N. Wild ${ }^{11}$, C. Wiebusch ${ }^{36}$, G. Wieczorek ${ }^{70}$, L. Wiencke ${ }^{84}$, B. Wilczyńska ${ }^{69}$, H. Wilczyński ${ }^{69}$, C. Wileman ${ }^{81}$, M.G. Winnick ${ }^{11}$, G. Wörner ${ }^{37}$, H. $\mathrm{Wu}^{32}$, B. Wundheiler ${ }^{2}$, T. Yamamoto ${ }^{96}{ }^{a}$, P. Younk $^{85}$, G. Yuan ${ }^{88}$, A. Yushkov ${ }^{48}$, E. Zas ${ }^{78}$, D. Zavrtanik ${ }^{73,72}$, M. Zavrtanik ${ }^{72,73}$, I. Zaw ${ }^{90}$, A. Zepeda ${ }^{60} b$, M. Ziolkowski ${ }^{42}$

${ }^{1}$ Centro Atómico Bariloche and Instituto Balseiro (CNEA-UNCuyo-CONICET), San Carlos de Bariloche, Argentina

${ }^{2}$ Centro Atómico Constituyentes (Comisión Nacional de Energía Atómica/CONICET/UTN-FRBA), Buenos Aires, Argentina

${ }^{3}$ Centro de Investigaciones en Láseres y Aplicaciones, CITEFA and CONICET, Argentina

${ }^{4}$ Departamento de Física, FCEyN, Universidad de Buenos Aires y CONICET, Argentina

${ }^{6}$ IFLP, Universidad Nacional de La Plata and CONICET, La Plata, Argentina

${ }^{7}$ Instituto de Astronomía y Física del Espacio (CONICET), Buenos Aires, Argentina

${ }^{8}$ Universidad Tecnológica Nacional, Facultad Regional Mendoza, (UTN-FRM), Mendoza, Argentina

${ }^{9}$ Pierre Auger Southern Observatory, Malargüe, Argentina

${ }^{10}$ Pierre Auger Southern Observatory and Comisión Nacional de Energía Atómica, Malargüe, Argentina

${ }^{11}$ University of Adelaide, Adelaide, S.A., Australia

12 Universidad Catolica de Bolivia, La Paz, Bolivia

${ }^{13}$ Universidad Mayor de San Andrés, Bolivia

${ }^{14}$ Centro Brasileiro de Pesquisas Fisicas, Rio de Janeiro, RJ, Brazil

${ }^{15}$ Pontifícia Universidade Católica, Rio de Janeiro, RJ, Brazil

16 Universidade de São Paulo, Instituto de Física, São Carlos, SP, Brazil

17 Universidade de São Paulo, Instituto de Física, São Paulo, SP, Brazil

18 Universidade Estadual de Campinas, IFGW, Campinas, SP, Brazil 
19 Universidade Estadual de Feira de Santana, Brazil

${ }^{20}$ Universidade Estadual do Sudoeste da Bahia, Vitoria da Conquista, BA, Brazil

${ }^{21}$ Universidade Federal da Bahia, Salvador, BA, Brazil

${ }^{22}$ Universidade Federal do ABC, Santo André, SP, Brazil

${ }^{23}$ Universidade Federal do Rio de Janeiro, Instituto de Física, Rio de Janeiro, RJ, Brazil

${ }^{24}$ Universidade Federal Fluminense, Instituto de Fisica, Niterói, RJ, Brazil

${ }^{26}$ Charles University, Faculty of Mathematics and Physics, Institute of

Particle and Nuclear Physics, Prague, Czech Republic

${ }^{27}$ Institute of Physics of the Academy of Sciences of the Czech Republic, Prague, Czech Republic

${ }^{28}$ Palacký University, Olomouc, Czech Republic

${ }^{30}$ Institut de Physique Nucléaire d'Orsay (IPNO), Université Paris 11, CNRS-IN2P3, Orsay, France

${ }^{31}$ Laboratoire AstroParticule et Cosmologie (APC), Université Paris 7, CNRS-IN2P3, Paris, France

${ }^{32}$ Laboratoire de l'Accélérateur Linéaire (LAL), Université Paris 11, CNRS-IN2P3, Orsay, France

${ }^{33}$ Laboratoire de Physique Nucléaire et de Hautes Energies (LPNHE),

Universités Paris 6 et Paris 7, CNRS-IN2P3, Paris Cedex 05, France

${ }^{34}$ Laboratoire de Physique Subatomique et de Cosmologie (LPSC), Université Joseph Fourier, INPG, CNRS-IN2P3, Grenoble, France

35 SUBATECH, CNRS-IN2P3, Nantes, France

${ }^{36}$ Bergische Universität Wuppertal, Wuppertal, Germany

37 Forschungszentrum Karlsruhe, Institut für Kernphysik, Karlsruhe, Germany

${ }^{38}$ Forschungszentrum Karlsruhe, Institut für Prozessdatenverarbeitung und Elektronik, Germany

39 Max-Planck-Institut für Radioastronomie, Bonn, Germany

${ }^{40}$ RWTH Aachen University, III. Physikalisches Institut A, Aachen, Germany

${ }^{41}$ Universität Karlsruhe (TH), Institut für Experimentelle Kernphysik

(IEKP), Karlsruhe, Germany

${ }^{42}$ Universität Siegen, Siegen, Germany

${ }^{44}$ Dipartimento di Fisica dell'Università and INFN, Genova, Italy

${ }^{45}$ Università dell'Aquila and INFN, L'Aquila, Italy

46 Università di Milano and Sezione INFN, Milan, Italy

${ }^{47}$ Dipartimento di Fisica dell'Università del Salento and Sezione INFN, Lecce, Italy

48 Università di Napoli "Federico II" and Sezione INFN, Napoli, Italy

${ }^{49}$ Università di Roma II "Tor Vergata" and Sezione INFN, Roma, Italy

${ }^{50}$ Università di Catania and Sezione INFN, Catania, Italy

${ }^{51}$ Università di Torino and Sezione INFN, Torino, Italy

${ }^{53}$ Istituto di Astrofisica Spaziale e Fisica Cosmica di Palermo (INAF),

Palermo, Italy and Sezione INFN, Catania, Italy

${ }^{54}$ Istituto di Fisica dello Spazio Interplanetario (INAF), Università di Torino and Sezione INFN, Torino, Italy 
55 INFN, Laboratori Nazionali del Gran Sasso, Assergi (L'Aquila), Italy

${ }^{56}$ Dipartimento di Ingegneria dell'Innovazione dell'Università del Salento and Sezione INFN, Lecce, Italy

57 INFN, Laboratori Nazionali del Sud, Catania, Italy

${ }^{59}$ Benemérita Universidad Autónoma de Puebla, Puebla, Mexico

${ }^{60}$ Centro de Investigación y de Estudios Avanzados del IPN (CINVESTAV), México, D.F., Mexico

${ }^{61}$ Instituto Nacional de Astrofisica, Optica y Electronica, Tonantzintla, Puebla, Mexico

${ }^{63}$ Universidad Michoacana de San Nicolas de Hidalgo, Morelia, Michoacan, Mexico

${ }^{64}$ Universidad Nacional Autonoma de Mexico, Mexico, D.F., Mexico

${ }^{65}$ IMAPP, Radboud University, Nijmegen, Netherlands

${ }^{66}$ Kernfysisch Versneller Instituut, University of Groningen, Groningen, Netherlands

67 NIKHEF, Amsterdam, Netherlands

68 ASTRON, Dwingeloo, Netherlands

${ }^{69}$ Institute of Nuclear Physics PAN, Krakow, Poland

${ }^{70}$ University of Łódź, Łódź, Poland

${ }^{71}$ LIP and Instituto Superior Técnico, Lisboa, Portugal

72 J. Stefan Institute, Ljubljana, Slovenia

${ }^{73}$ Laboratory for Astroparticle Physics, University of Nova Gorica, Slovenia

${ }^{74}$ Instituto de Física Corpuscular, CSIC-Universitat de València, Valencia, Spain

${ }^{75}$ Universidad Complutense de Madrid, Madrid, Spain

${ }^{76}$ Universidad de Alcalá, Alcalá de Henares (Madrid), Spain

77 Universidad de Granada \& C.A.F.P.E., Granada, Spain

${ }^{78}$ Universidad de Santiago de Compostela, Spain

${ }^{79}$ Rudolf Peierls Centre for Theoretical Physics, University of Oxford, Oxford, United Kingdom

${ }^{81}$ School of Physics and Astronomy, University of Leeds, United Kingdom

82 Argonne National Laboratory, Argonne, IL, USA

${ }^{83}$ Case Western Reserve University, Cleveland, OH, USA

${ }^{84}$ Colorado School of Mines, Golden, CO, USA

${ }^{85}$ Colorado State University, Fort Collins, CO, USA

${ }^{86}$ Colorado State University, Pueblo, CO, USA

${ }^{87}$ Fermilab, Batavia, IL, USA

${ }^{88}$ Louisiana State University, Baton Rouge, LA, USA

${ }^{89}$ Michigan Technological University, Houghton, MI, USA

${ }^{90}$ New York University, New York, NY, USA

${ }^{91}$ Northeastern University, Boston, MA, USA

92 Ohio State University, Columbus, OH, USA

${ }^{93}$ Pennsylvania State University, University Park, PA, USA

94 Southern University, Baton Rouge, LA, USA

${ }^{95}$ University of California, Los Angeles, CA, USA

${ }^{96}$ University of Chicago, Enrico Fermi Institute, Chicago, IL, USA 
${ }^{98}$ University of Hawaii, Honolulu, HI, USA

100 University of Nebraska, Lincoln, NE, USA

${ }^{101}$ University of New Mexico, Albuquerque, NM, USA

102 University of Pennsylvania, Philadelphia, PA, USA

${ }^{103}$ University of Wisconsin, Madison, WI, USA

${ }^{104}$ University of Wisconsin, Milwaukee, WI, USA

105 Institute for Nuclear Science and Technology (INST), Hanoi, Vietnam

(†) Deceased

(a) at Konan University, Kobe, Japan

(b) On leave of absence at the Instituto Nacional de Astrofisica, Optica y Electronica

(c) at Caltech, Pasadena, USA

\begin{abstract}
The Pierre Auger Observatory is a hybrid detector for ultra-high energy cosmic rays. It combines a surface array to measure secondary particles at ground level together with a fluorescence detector to measure the development of air showers in the atmosphere above the array. The fluorescence detector comprises 24 large telescopes specialized for measuring the nitrogen fluorescence caused by charged particles of cosmic ray air showers. In this paper we describe the components of the fluorescence detector including its optical system, the design of the camera, the electronics, and the systems for relative and absolute calibration. We also discuss the operation and the monitoring of the detector. Finally, we evaluate the detector performance and precision of shower reconstructions.
\end{abstract}

Key words: cosmic rays, fluorescence detector PACS: 96.40.-z, 96.40.Pq, 98.70.Sa 


\section{Introduction}

The hybrid detector of the Pierre Auger Observatory [1] consists of 1600 surface stations - water Cherenkov tanks and their associated electronics - and 24 air fluorescence telescopes. The Observatory is located outside the city of Malargüe, Argentina ( $69^{\circ} \mathrm{W}, 35^{\circ} \mathrm{S}, 1400 \mathrm{~m}$ a.s.l.) and the detector layout is shown in Fig. 1. Details of the construction, deployment and maintenance of the array of surface detectors are described elsewhere [2]. In this paper we will concentrate on details of the fluorescence detector and its performance.

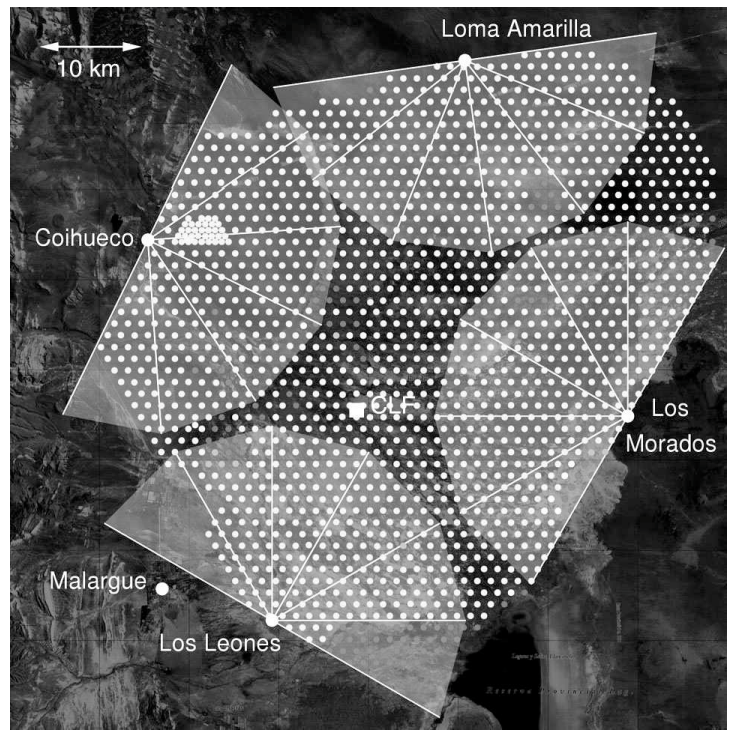

Figure 1: Status of the Pierre Auger Observatory as of March 2009. Gray dots show the positions of surface detector stations, lighter gray shades indicate deployed detectors, while dark gray defines empty positions. Light gray segments indicate the fields of view of 24 fluorescence telescopes which are located in four buildings on the perimeter of the surface array. Also shown is a partially completed infill array near the Coihueco station and the position of the Central Laser Facility (CLF, indicated by a white square). The description of the CLF and also the description of all other atmospheric monitoring instruments of the Pierre Auger Observatory is available in [3].

The detection of ultra-high energy $\left(\gtrsim 10^{18} \mathrm{eV}\right)$ cosmic rays using nitrogen fluorescence emission induced by extensive air showers is a well established technique, used previously by the Fly's Eye [4] and HiRes [5] experiments. It is used also for the Telescope Array [6] project that is currently under construction, and it has been proposed for the satellite-based EUSO and OWL projects.

Charged particles generated during the development of extensive air showers excite atmospheric nitrogen molecules, and these molecules then emit fluorescence light in the $\sim 300-430 \mathrm{~nm}$ range. The number of emitted fluorescence photons is proportional to the energy deposited in the atmosphere due to electromagnetic energy losses by the charged particles. By measuring the rate 


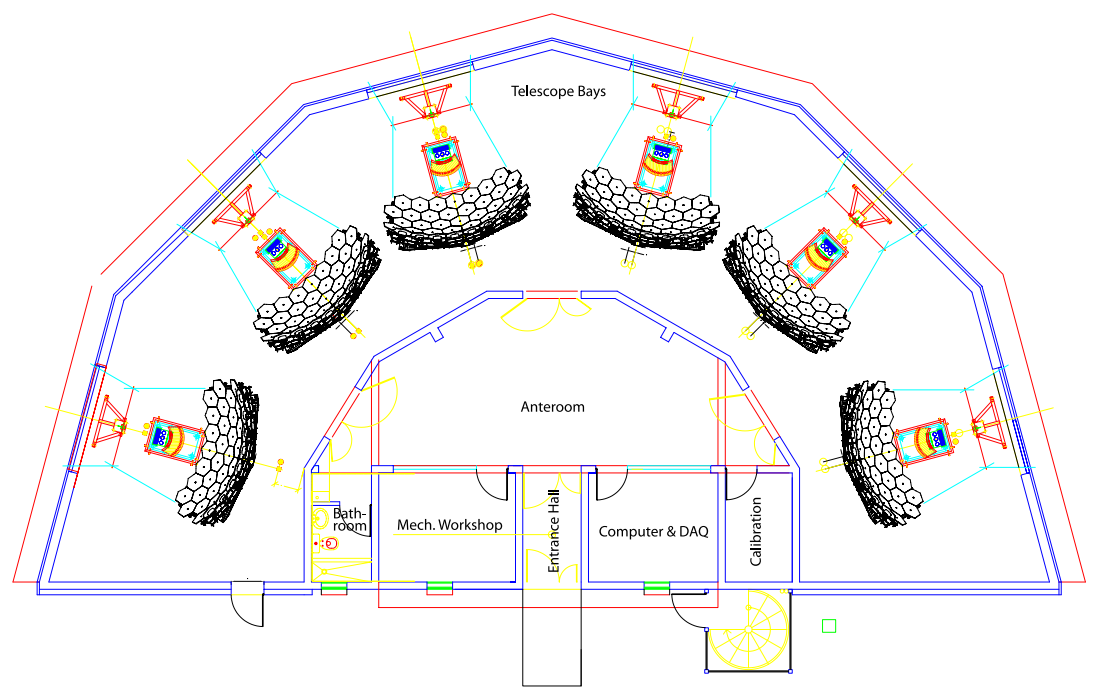

Figure 2: Schematic layout of the building with six fluorescence telescopes.

of fluorescence emission as a function of atmospheric slant depth $X$, an air fluorescence detector measures the longitudinal development profile $\frac{d E}{d X}(X)$ of the air shower. The integral of this profile gives the total energy dissipated electromagnetically, which is approximately $90 \%$ of the total energy of the primary cosmic ray.

For any waveband, the fluorescence yield is defined as the number of photons emitted in that band per unit of energy loss by charged particles. The absolute fluorescence yield in air at $293 \mathrm{~K}$ and $1013 \mathrm{hPa}$ from the $337 \mathrm{~nm}$ fluorescence band is $5.05 \pm 0.71$ photons/MeV of energy deposited, as measured in [7]. The wavelength dependence of the yield has been described e.g. in [8]. Since a typical cosmic ray shower spans over $10 \mathrm{~km}$ in altitude, it is important to stress that due to collisional quenching effects the fluorescence yield is also dependent on pressure, temperature and humidity of the air.

The fluorescence detector (FD) comprises four observation sites - Los Leones, Los Morados, Loma Amarilla, and Coihueco - located atop small elevations on the perimeter of the SD array. Six independent telescopes, each with field of view of $30^{\circ} \times 30^{\circ}$ in azimuth and elevation, are located in each FD site. The telescopes face towards the interior of the array so that the combination of the six telescopes provides $180^{\circ}$ coverage in azimuth. Figure 2 shows the arrangement of the telescopes inside an observation site.

Figure 3 depicts an individual FD telescope. The telescope is housed in a clean climate-controlled building. Nitrogen fluorescence light enters through a large UV-passing filter window and a Schmidt optics corrector ring. The light is focused by a 10 square meter mirror onto a camera of 440 pixels with photomultiplier light sensors. Light pulses in the pixels are digitized every 100 
nanoseconds, and a hierarchy of trigger levels culminates in the detection and recording of cosmic ray air showers.

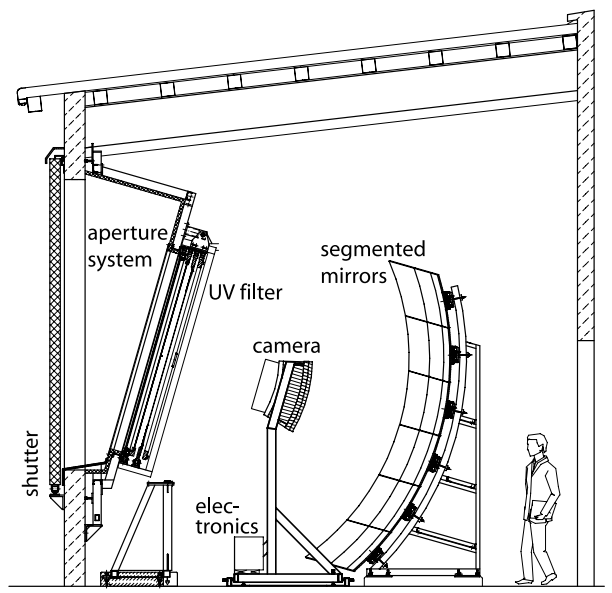

Figure 3: Schematic view of a fluorescence telescope of the Pierre Auger Observatory.

This paper is organized as follows. In section 2 we describe the components of the optical system of an individual telescope, and in section 3 we focus on the telescope camera. The electronics of a fluorescence telescope and the data acquisition system (DAQ) of an FD station are described in section 4. The details of the calibration hardware and methods are given in section 5 and the performance, operation and monitoring of the fluorescence detector are explained in section 6. Finally, in section 7 we concentrate on the basics of shower reconstruction using the measured fluorescence signal, and in section 8 we summarize.

\section{Optical system}

The basic elements of the optical system in each FD telescope are a filter at the entrance window, a circular aperture, a corrector ring, a mirror and a camera with photomultipliers. The geometrical layout of the components is depicted in Fig. 4 .

The window is an optical filter made of Schott MUG-6 glass [10]. This absorbs visible light while transmitting UV photons up to $410 \mathrm{~nm}$ wavelength, which includes almost all of the nitrogen fluorescence spectrum. Without the filter window, the fluorescence signals would be lost in the noise of visible photons.

The aperture, the corrector ring, the mirror, and the PMT camera constitute a modified Schmidt camera design that partially corrects spherical aberration and eliminates coma aberration. The size of the aperture is optimized to keep 
the spot sizd 1 due to spherical aberration within a diameter of $15 \mathrm{~mm}$, i.e. $90 \%$ of the light from a distant point source located anywhere within the $30^{\circ} \times 30^{\circ}$ FOV of a camera falls into a circle of this diameter. This corresponds to an angular spread of $0.5^{\circ}$. In comparison, the FOV of a single camera pixel is $1.5^{\circ}$. The light distribution within the spot is described by the point spread function (PSF) shown in Fig. 5 .

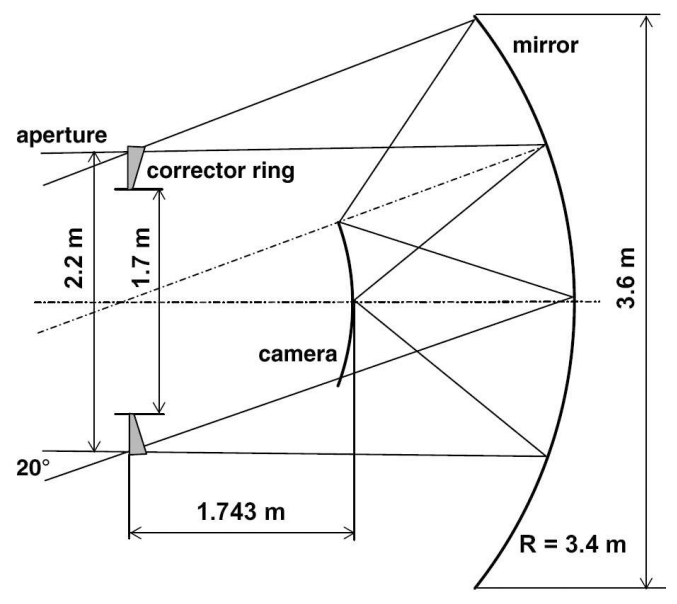

Figure 4: Geometrical parameters of the FD telescopes.

The schematic view of the spot size diagrams over the whole FOV is shown in Fig. 6] where the rows correspond to viewing angles $0^{\circ}, 10^{\circ}, 15^{\circ}$ and $20^{\circ}$. The columns corresponds to different displacements of the camera off the focal plane, by changing the camera-mirror distance from $-5 \mathrm{~mm}$ to $+5 \mathrm{~mm}$ with respect to the nominal separation. The central position is located at a distance of $1657 \mathrm{~mm}$ from the primary mirror. The asymmetric shape of some spots is due to vignetting and camera shadow. This picture also shows the sensitivity of the telescope PSF to the precision of the adjustment of the distance between mirror and camera.

\subsection{Segmented mirror}

Due to the large area of the primary mirror $\left(\sim 13 \mathrm{~m}^{2}\right)$, the mirror is segmented to reduce the cost and weight of the optical system. Two segmentation configurations are used: first, a tessellation of 36 rectangular anodized aluminum mirrors of three different sizes; and second, a structure of 60 hexagonal glass mirrors (of four shapes and sizes) with vacuum-deposited

\footnotetext{
${ }^{1}$ The image of the point source at infinity on the focal surface of the optical system is commonly called the "spot" in optics, but it may be better known as a "point spread function". The size of the spot characterizes the quality of the optical system.
} 

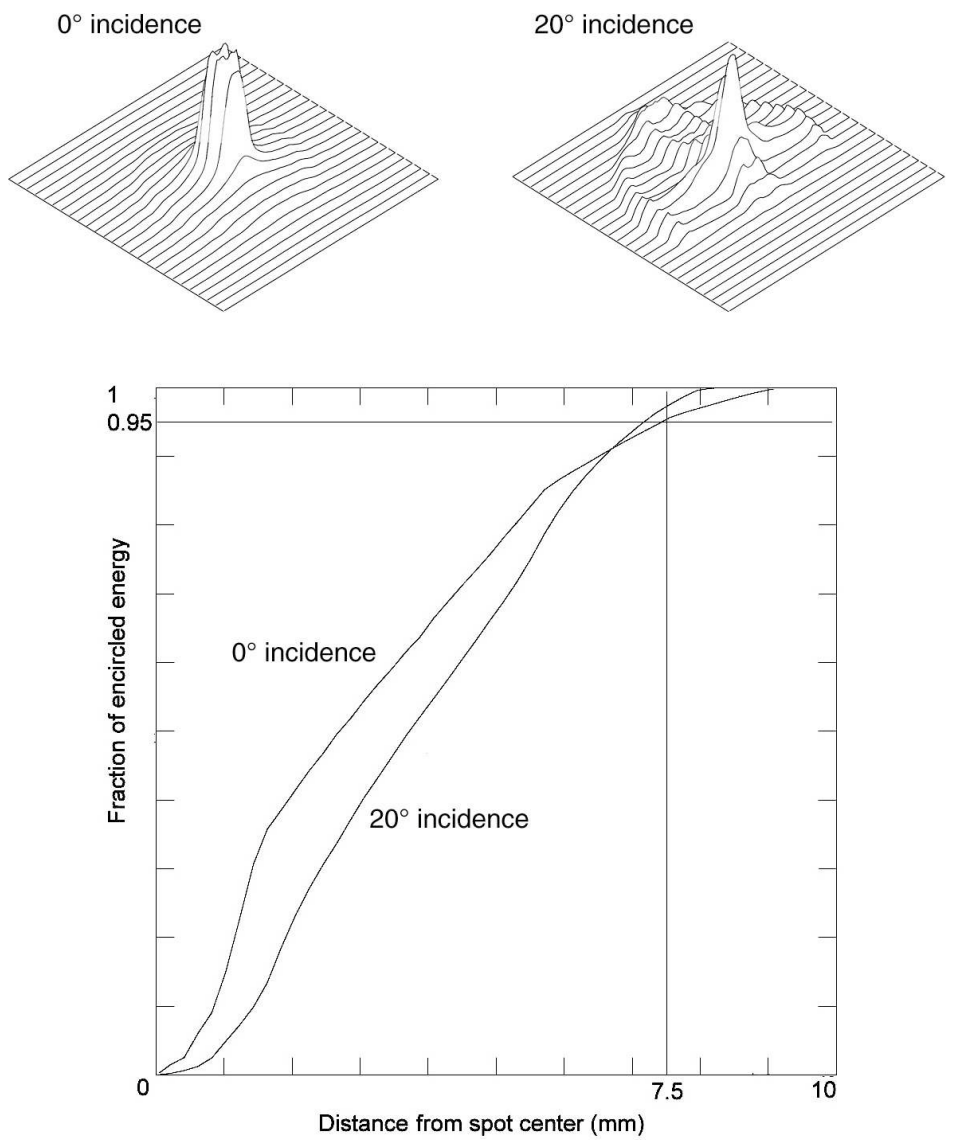

Figure 5: Top: The simulated 3D distribution of the light intensity for spots on the optical axis (left) and close to the camera corner (right; distance from the optical axis $=20^{\circ}$ ). The size of the imaged area is $20 \times 20 \mathrm{~mm}$. Bottom: Fraction of encircled energy as a function of spot diameter for the spot on the optical axis (upper curve; angular distance from the optical axis $=0^{\circ}$; this curve corresponds to the distribution shown top left) and for the spot close to the camera corner (lower curve; angular distance from the optical axis $=20^{\circ}$; this curve corresponds to the distribution shown top right).

reflective coatings. In both cases, all mirror segments have a spherical inner radius of $3400 \mathrm{~mm}$, allowing possible deviations up to $3420 \mathrm{~mm}$.

The 12 telescopes at the Los Leones and Los Morados sites use aluminum mirrors. The mirror elements were produced from $20 \mathrm{~mm}$-thick cast aluminum blocks, and were milled to the required spherical inner radius using a diamond milling technique. After the initial milling, the reflective surface was created by gluing a $2 \mathrm{~mm}$ sheet of $\mathrm{AlMgSiO}_{5}$ alloy onto the inner surface of each element. The sheets were attached to the aluminum block at elevated temperature and 


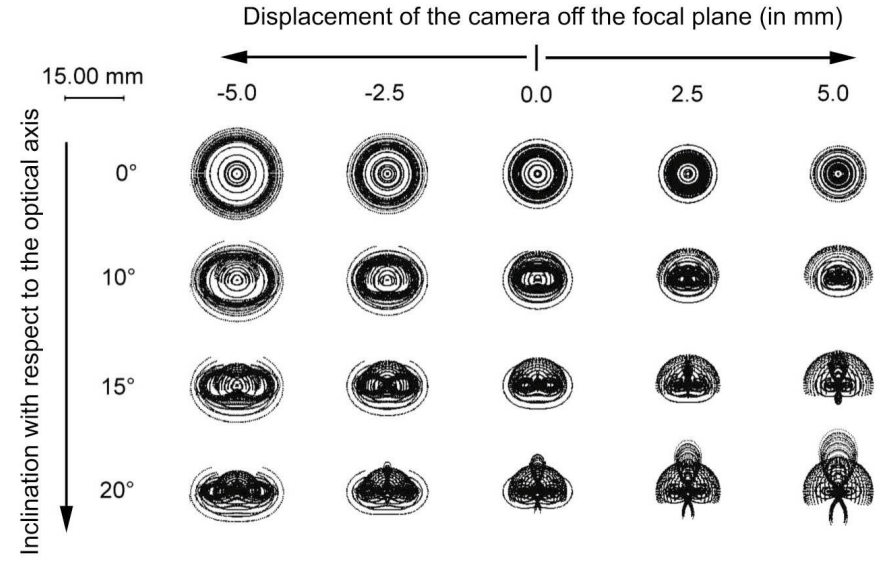

Figure 6: Spot diagrams of the telescope.
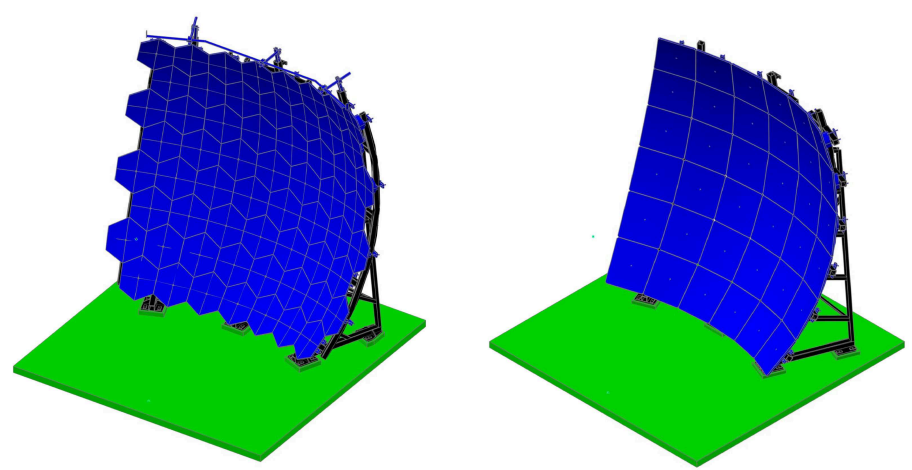

Figure 7: Two different segmentation configurations of the telescope mirror: left: 36 rectangular anodized aluminum mirror segments; right: 60 hexagonal glass mirror segments.

pressure to achieve high stability. The material used is well-suited for highprecision diamond milling, and allows the final mirror surface to achieve a roughness below $10 \mathrm{~nm}$. Finally, a $90 \mathrm{~nm}$ thick aluminum-oxide layer was applied to the surface by chemical anodization to provide additional protection.

The remaining 12 telescopes in the buildings at Coihueco and Loma Amarilla use mirrors with glass segments. The thickness of the glass is $12 \mathrm{~mm}$, and it is composed of SIMAX, a borosilicate glass of the PYREX type. The choice of ultra-thin, lightweight glass was motivated by the need to maintain the optical stability of the segments at different temperatures and for different inclinations of the individual segments. SIMAX is suitable for machine grinding and polishing, and has good mechanical and thermal stability. 
The reflective layer on the glass mirror segments is composed of Al layer, with thickness of $90 \mathrm{~nm}$, covered by one $\mathrm{SiO}_{2}$ layer with thickness of $110 \mathrm{~nm}$. The aluminum is used for its high reflectivity, and the silica for its high mechanical resistance. The reflectivity has been measured at several points of each segment, and the average reflectivity at $\lambda=370 \mathrm{~nm}$ exceeds $90 \%$. The surface roughness has been measured mechanically and optically, and has an RMS of less than $10 \mathrm{~nm}$.

In the glass mirrors, the mirror segments are grouped by their curvature radii to minimize the radius deviations in each telescope. The curvature and the shape of the reflective surface of the segments are measured and controlled using standard Ronchi and/or Hartmann tests [11].

The alignment of the mirror segments is accomplished by directing a laser onto each segment. The laser is mounted on the center of curvature of the full mirror. The laser beam approximates a point source, and each segment is adjusted such that the beam is reflected to a common point (the center of the laser aperture). This procedure is used to adjust all the mirror segments and match the full mirror to the required spherical shape, with the center of curvature aligned with the optical axis of the telescope.

\subsection{Corrector ring}

A novel solution of the optical system with a "corrector ring" was designed to keep the advantage of a large aperture of the Schmidt system, and simultaneously simplify the production of the element, minimize its weight and cost, while maintaining the spot size within the limits of aforementioned design specification [12]. The aperture area of the telescope with the corrector ring is almost doubled with respect to the optical system without any correcting element. The analysis of real shower data [13] has compared the performances of FD optical systems with and without corrector ring, and has verified that the corrector ring enhances the FD aperture by a factor of $\sim 2$.

The corrector ring is the circumferential part of the corrector plate of a classical Schmidt camera with one planar side and the other with an aspheric shape corresponding to a $6^{\text {th }}$-order polynomial curve. Such a surface is difficult to manufacture and therefore some optimizations were adopted to simplify the lens production. Eventually, a spherical approximation specially designed for the fluorescence detector was chosen to fulfill both price and performance requirements (see Fig. 8) . The simplified corrector ring is located at the aperture and covers the annulus between radii of $0.85 \mathrm{~m}$ and $1.1 \mathrm{~m}$.

Since the rings have an external diameter of $2.2 \mathrm{~m}$, their manufacture and transportation to the site in a single piece would have been very difficult. Therefore, each lens was divided into 24 segments. The size and profile of one segment is shown in Fig. 9. The production of the segments has been performed by Schwantz Ltd 14] after assembling a machine with a circular base to hold

\footnotetext{
${ }^{2}$ Test measurements without corrector ring were realized on several FD telescopes prior to corrector ring installation.
} 


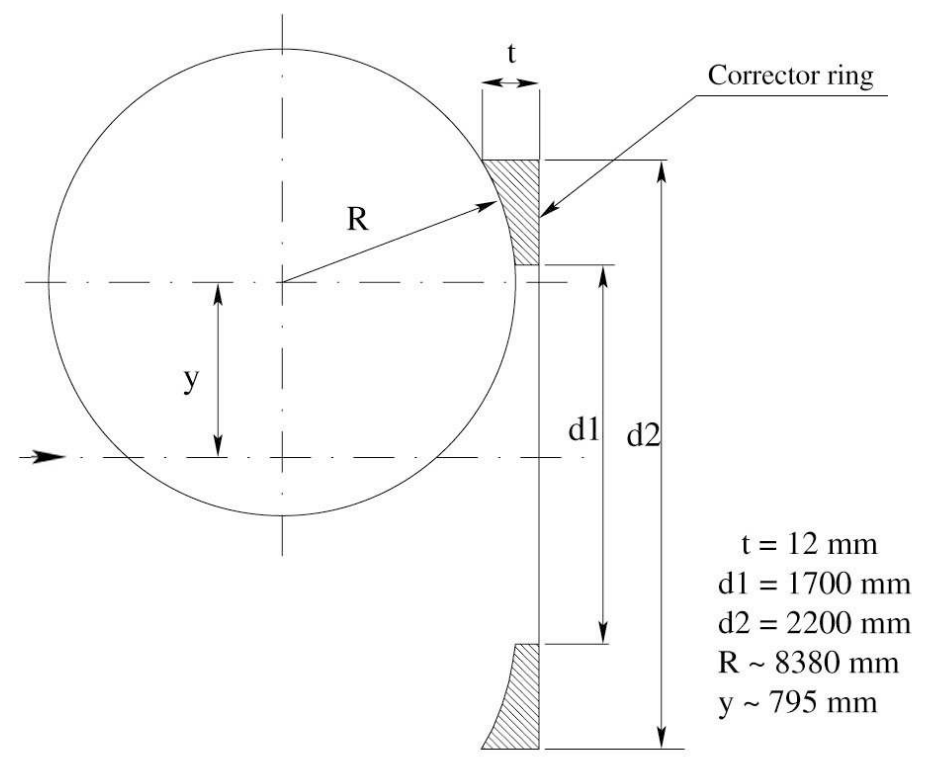

Figure 8: Corrector ring profile using a spherical approximation, the optical axis of the telescope is identified by the dash-dotted line with an arrow on the left and $d 2$ is the diameter of the aperture.

the segments, and a disk with diamond abrasive cylinders for the grinding of the glass (BK7 glass from Schott [10]) to the desired profile. After production of each ring, its segments were tested for proper shape. To scan the ring profiles, a laser beam was pointed towards the curved and flat surfaces and the positions of the reflected light from each surface were measured [12].

\subsection{Simulation of the optical system}

To evaluate the overall optical performance of the detector and to confirm the theoretical expectations, a dedicated complete simulation of the optical system was developed using Geant4 [15, 16], a Monte Carlo toolkit for the simulation of radiation and light propagation. The tracking of optical photons includes refraction and reflection at medium boundaries, Rayleigh scattering and bulk absorption. The optical properties of a medium, such as refractive index, absorption length, and reflectivity coefficients, can be expressed as functions of the wavelength. The application includes a detailed description of the different detector elements - UV filter, corrector lens, mirror and camera. The optical properties of all materials, such as the absorption length and the refractive index, were implemented as a function of wavelength.

The simulation confirms that all design specifications of the FD optical 


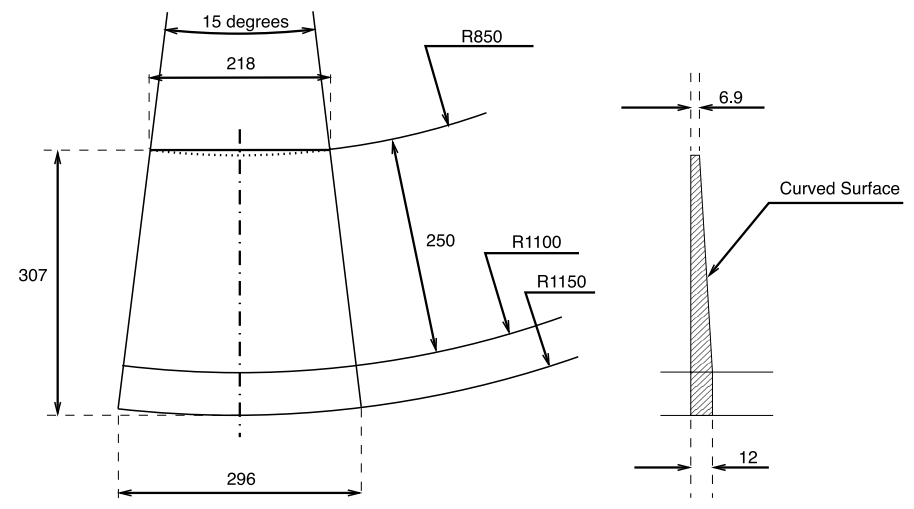

Figure 9: Technical drawing of one corrector ring segment. The dimensions are given in millimeters.

system are met, i.e. even in the corners of the camera $90 \%$ of the light from a distant point source is concentrated within a diameter of $15 \mathrm{~mm}$.

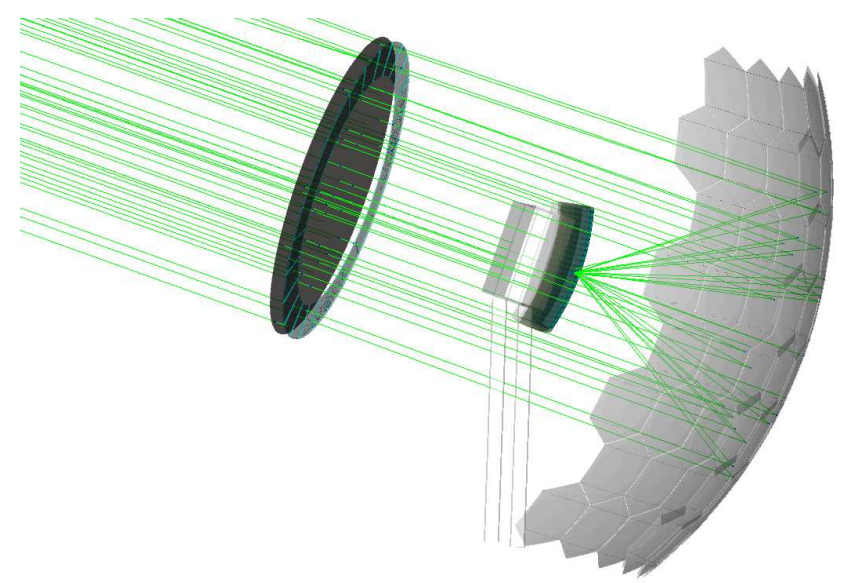

Figure 10: Ray tracing simulation of the optical system of the telescope made using Geant4.

\section{Telescope camera}

The camera [17] is the sensitive element of a telescope. It is composed of a matrix of 440 pixels located on the focal surface of the telescope. The camera pixels are arranged in a matrix of 22 rows by 20 columns (Fig. 11k). The corresponding field of view is of $30^{\circ}$ in azimuth (full acceptance of one row) and $28.1^{\circ}$ in elevation (full acceptance of one column). 
A cosmic ray shower is imaged on the camera as a line of activated pixels having a track-like geometrical pattern and also a clear time sequence. Each pixel is realized by a photomultiplier with a light collector.

\subsection{Geometry}

The pixel array lies on the focal surface of the optical system, which is a sphere of $1.743 \mathrm{~m}$ radius. The pixels are hexagonal with a side to side distance of $45.6 \mathrm{~mm}$, corresponding to an angular size of $1.5^{\circ}$. The pixel centers are placed on the spherical surface following the procedure outlined in Fig. 11, where we define $\Delta \theta=1.5^{\circ}$ and $\Delta \phi=0.866^{\circ}$. The first center is placed at $+\Delta \theta / 2$ with respect to the telescope axis, which is taken as the $z$ axis in Fig. [1]. The other pixel centers are obtained with increasing (or decreasing) $\Delta \theta$ steps. In this manner, a row of twenty pixels (corresponding to $30^{\circ}$ in azimuth) is built.

The following row of pixels is obtained by a rotation of $\Delta \phi$ around the $x$ axis. To produce the correct staggering between rows, the pixel centers are moved by $\Delta \theta / 2$ with respect to their positions in the previous row.

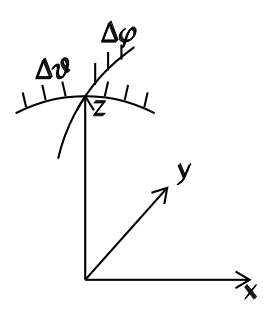

a)

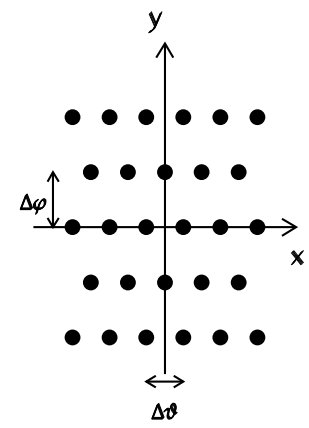

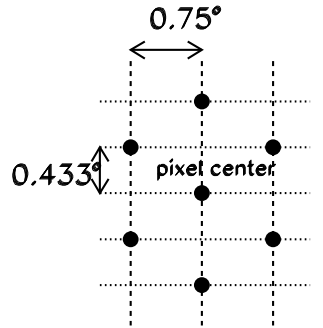

b)

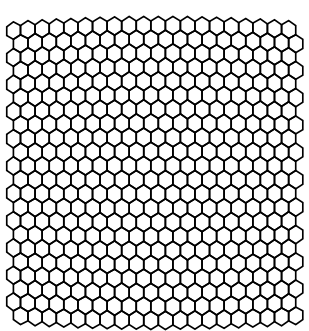

c)

Figure 11: Geometrical construction of the FD camera; a) pixel centers are placed on the spherical surface in steps of $\Delta \theta=1.5^{\circ}$ and $\Delta \phi=0.866^{\circ}$, b) positioning of the pixel vertices around the pixel center, c) the camera with 440 pixels arranged in a $22 \times 20$ matrix.

Once the pixel centers have been defined, each pixel hexagon is determined by positioning six vertices around the center. The angular positions of the vertices are obtained by moving in steps of $\Delta \theta / 2$ and $\Delta \phi / 3$ with respect to the 
pixel center, as depicted in Fig. [11b. Equal steps in angle produce different linear dimensions depending on the pixel position on the spherical surface. Therefore, the pixels are not exactly regular hexagons, but their size varies over the focal surface by $3.5 \%$ at most. Differences in the side length are smaller than $1 \mathrm{~mm}$, and are taken into account in the design of the light collectors (see section 3.3) and in the analysis.

\subsection{Mechanics}

The camera body was produced from a single aluminum block by a programmable milling machine. It consists of a plate of $60 \mathrm{~mm}$ uniform thickness and approximately rectangular shape $(930 \mathrm{~mm}$ horizontal $\times 860 \mathrm{~mm}$ vertical), with spherical outer and inner surfaces. The outer radius of curvature is $1701 \mathrm{~mm}$, while the inner radius is $1641 \mathrm{~mm}$. Photomultiplier tubes are positioned inside $40 \mathrm{~mm}$ diameter holes drilled through the plate on the locations of the pixel centers. Small holes in the camera body at the pixel vertices are used to secure the light collectors in place. A picture of the camera body is shown in Fig. 12 .

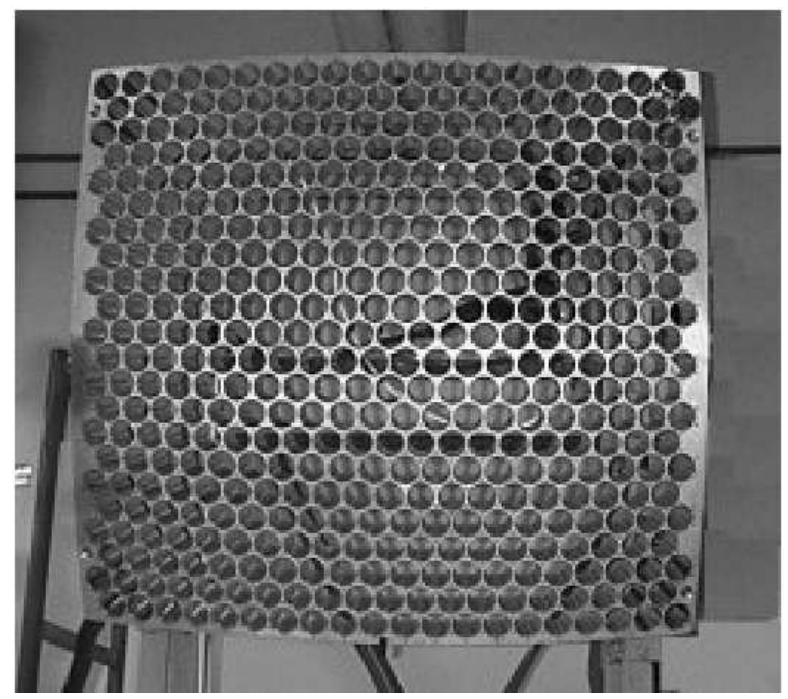

Figure 12: Picture of the camera body.

Within a finished telescope, the camera body is held in place by a simple and robust two-leg steel support made of $5 \mathrm{~cm}$ wide $\mathrm{C}$-shaped steel beams. The obscuration of the mirror FOV due to the camera support is less than one-tenth of that of the camera. Power and signal cables run inside the C-shaped legs of the support without producing additional obscuration. 


\subsubsection{Mechanical precision and alignment}

To align an FD telescope, the pixel surface must be placed at the correct longitudinal distance from the center of curvature of the mirror, while the camera body should be centered on and perpendicular to the telescope axis, with the top and bottom sides of the camera parallel to the ground. Ray tracing computations have shown that the spot size increases by about $10 \%$ when the longitudinal distance from the center of curvature changes by $\pm 2 \mathrm{~mm}$. Therefore, the accuracy on the longitudinal position of the pixels on the focal surface should be better than $\pm 2 \mathrm{~mm}$.

The intrinsic accuracy of the rigid metal frame of of the camera body is very good, at the level of $\pm 0.1 \mathrm{~mm}$. The point-to-point internal accuracy of the pixels on the camera body is at the level of $\pm 1 \mathrm{~mm}$ in both the longitudinal and transverse directions, due to the positioning of the photomultipliers and of the light concentrators on the rigid frame of the camera body.

The fluorescence buildings are surveyed by standard topographic methods and for each bay two accurately determined reference points are marked on the floor defining a line which corresponds to the azimuth of the telescope axis. The center of the camera is placed in the vertical plane containing this line and at the right nominal height. The camera is then aligned horizontally using a digital level-inclinometer with a precision better than $0.05^{\circ}$ which is placed on the top of the camera body. Once the center of the camera is correctly positioned and the camera is horizontal, the right orientation is achieved measuring the distance of the four corners of the camera body to the mechanical reference point which is located at the mirror center. These measurements are done with a commercial laser distance-meter with a precision of $\pm 1 \mathrm{~mm}$ mounted on the mirror center. The alignment procedure should provide a positioning with space accuracy at the level of $\pm 1 \mathrm{~mm}$ and angular precision at the level of one millirad i.e. better that $0.1^{\circ}$.

Measurements of the image of bright stars on the camera focal surface have verified that the alignment procedure for mirror elements and camera body meet the design specifications [18]. The precision of the absolute pointing of the telescopes has been checked to within an accuracy of $0.1^{\circ}$.

\subsection{The pixel array}

The hexagonal photomultiplier tube (PMT), model XP3062 manufactured by Photonis [19] is used to instrument the camera. Although their hexagonal shape represents the best approximation to the pixel geometry, a significant amount of insensitive area is nevertheless present between the photocathodes. In fact, some space between the PMTs is needed for safe mechanical packaging on the focal surface; moreover, the effective cathode area is smaller than the area delimited by the PMT glass envelope. To maximize light collection and guarantee a sharp transition between adjacent pixels, the hexagonal PMTs are complemented by light collectors.

The basic element for the pixel light collector is a Mercedes star, with three arms oriented $120^{\circ}$ apart, which is positioned on each pixel vertex. Six Mercedes 
stars collect the light for a given pixel. The geometrical structure of the light collector for one pixel is shown in Fig[13,

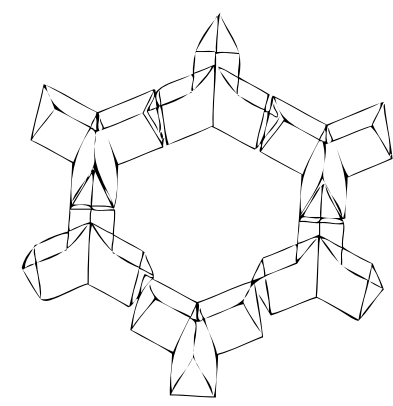

a)

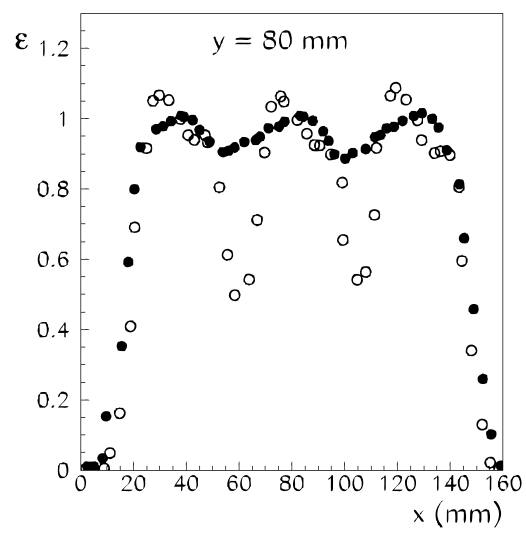

b)

Figure 13: a) Six Mercedes stars positioned in order to form a pixel. Each Mercedes star has three arms oriented $120^{\circ}$ apart. In the drawing the bottom Mercedes is slightly displaced for clarity. b) Measurement of the light collection efficiency, with a light spot moved along a line passing over three adjacent pixels. The full (open) dots represent the measurements performed with (without) Mercedes stars.

The Mercedes stars are made of plastic material covered by aluminized Mylar ${ }^{\mathrm{TM}}$ foils. The arm length is approximately half of the pixel side length. The arm section is an equilateral triangle. The base length of $9.2 \mathrm{~mm}$ is designed to cover the insensitive space due to photocathode inefficiency $(\approx 2 \mathrm{~mm}$ for each adjacent PMT) plus the maximum space between the glass sides of the PMTs (of the order of $5 \mathrm{~mm}$ ). The triangle height is $18 \mathrm{~mm}$, and the corresponding angle at the vertex is $14.3^{\circ}$.

Each Mercedes star is held by a bar, about $10 \mathrm{~cm}$ long, which is inserted into a $3.2 \mathrm{~mm}$ hole located on the position of the pixel vertices and kept in place by a small O-ring. The geometry of the light collectors was designed on the basis of the optical system properties. The range of angles of incidence for the rays on the camera is in the interval between approximately $10^{\circ}$ to $35^{\circ}$, as determined by the shadow of the camera and the aperture of the diaphragm. Note that the pixels are defined on the focal surface at $1743 \mathrm{~mm}$ from the center of curvature where the top edges of the Mercedes divide one pixel from its neighbors. The PMT cathodes are recessed behind that focal surface by $18 \mathrm{~mm}$.

Monte Carlo ray tracing has shown that the light collection efficiency, averaged over the camera, is $94 \%$, assuming a reflectivity of $85 \%$ for the aluminized Mylar foils of the light collector surface. Without the light collectors, the collection efficiency decreases to $70 \%$.

The Monte Carlo simulation was experimentally checked using a specially designed light diffusing cylinder with an exit hole for light rays having the same 
size as the spot produced by the telescope optics. The distribution of the angles of the light rays from this optical device is similar to that produced by the telescopes. Measurements were made with and without the light collectors. Results of a scan moving the light spot over the camera are shown in Fig. $13 \mathrm{~b}$. Without light collectors, a significant loss of light at the pixel borders was observed. When installed, the light collectors efficiently recuperate the light loss. From these measurements, the light collection efficiency averaged over the camera focal surface was found to be $93 \%$.

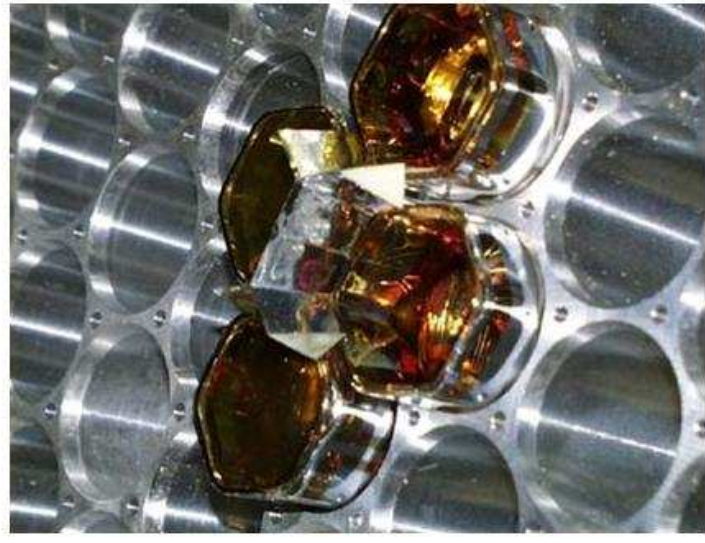

a)

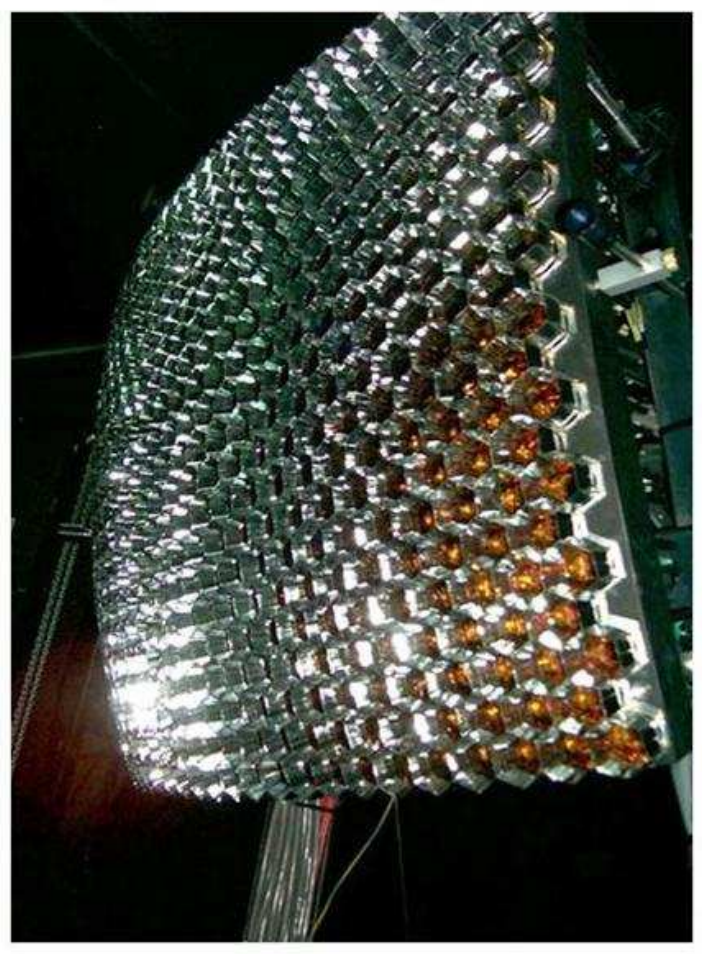

b)

Figure 14: a) Detail of the camera body with four PMTs mounted together with two Mercedes stars. The large holes to insert the PMTs and the small ones to mount the Mercedes are visible. b) Picture of a camera completely assembled with all PMTs and light collectors in place.

A picture of a small section of the camera and a full picture of the camera completely assembled are shown in Fig. 14

\subsection{The photomultiplier unit}

The XP3062 photomultiplier [19] is an 8-stage PMT with a hexagonal window (40 $\mathrm{mm}$ side to side). It is manufactured with a standard bialkaline 
photocathode with quantum efficiency of about $25 \%$ in the wavelength range $350-400 \mathrm{~nm}$. The nominal gain for standard operation of the FD is set to $5 \times 10^{4}$.

The PMT high voltage (HV) is provided by a HV-divider chain which forms a single physical unit together with the signal driver circuitry. This unit, called head electronics (HE), consists of three coaxially interconnected printed circuits boards (PCBs): the bias PCB (innermost one), a laser-trimmed hybrid technology driver circuit (intermediate), and the interface PCB (outermost). The innermost and outermost PCBs are two-sided and of circular shape (32 $\mathrm{mm}$ diameter), and are interconnected using high reliability pin connectors. The HE units were manufactured by Intratec-Elbau (Berlin, Germany) 22] and are soldered to the flying leads of the PMT. To ensure central mounting of the HE with respect to the symmetry axis of the PMT, and to improve mechanical rigidity, a specially-designed plastic structure has been introduced between the glass tube and a central guidance hole left in the innermost PCB of the HE. A PMT with the attached head electronics is shown in Fig. 15.

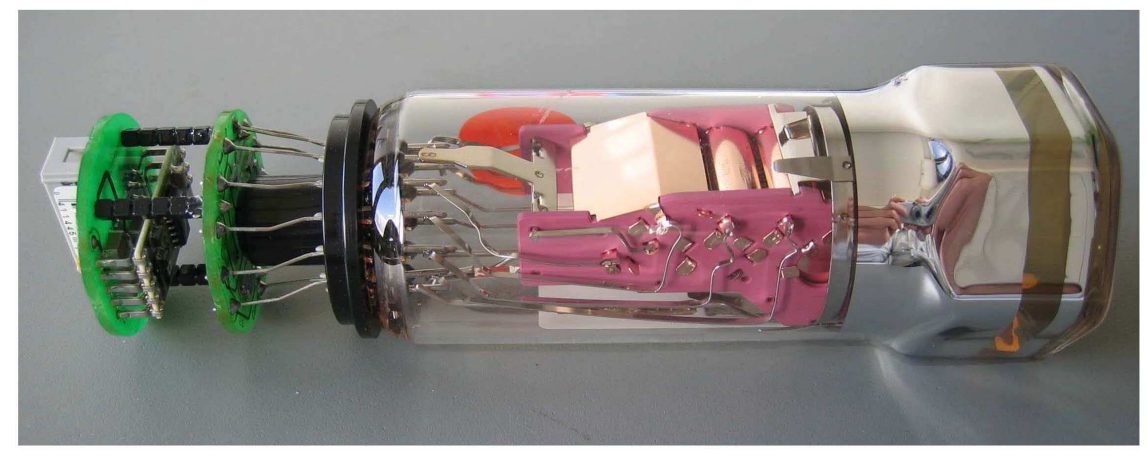

Figure 15: Picture of a PMT unit.

The HV divider keeps the PMT photocathode at ground and supplies positive HV to the anode. To dissipate minimum power in the dense package of $\mathrm{HE}$ units, the bleeder is operated at currents of less than $170 \mu \mathrm{A}$. Stabilization of the HV potential for large pulses and in presence of a relatively strong light background is thus realized by employing an active network 20] that uses bipolar transistors in the last three stages of the PMT. This is necessary for a fluorescence telescope, which is exposed to the dark sky background and in some cases also to a fraction of moon light. The normal dark sky background induces an anode current of about $0.8 \mu \mathrm{A}$. The active divider ensures that the gain shift due to the divider chain is less than $1 \%$ for anode currents up to about $10 \mu \mathrm{A}$.

The driver located on the intermediate ceramics board of the HE receives the AC-coupled anode signal through a differential input. The anode pulse flows through a load resistor and reaches one leg of the differential input of the line 
driver, while the other leg only picks up the common-mode noise. A commonmode rejection ratio of $28 \mathrm{~dB}$ is obtained between $1-100 \mathrm{kHz}$. The integrated laser-trimmed hybrid circuit supplies a balanced output matching the $120 \Omega$ characteristic impedance of the twisted pair cable connecting the HE to the front-end analog board of the readout electronics (see section 4.1.1).

Extensive qualification and acceptance tests 21] were performed on the full PMT unit, i.e. the PMT with the HE attached, using automatic test systems. Among the tests were measurements of the HV dependence of the gain, which are needed for a proper grouping of the PMTs with similar gain. The relative photocathode sensitivity at different wavelengths, the linearity of the PMT unit, and the photocathode uniformity were also measured. Before and after installation, the relevant information for each individual PMT unit is collected into a database which tracks the PMT characteristics, including calibration measurements performed in situ.

To reduce the cost of the power supplies, the photomultipliers of each camera are organized into ten groups of 44 units. Each group has similar gain characteristics, and is powered by a single HV channel. The spread of the gains within a group is about $\pm 10 \%$.

Cables are distributed to the PMT electronics through distribution boards positioned behind the camera and within its shadow, i.e., without causing additional obscuration. These boards serve groups of 44 PMTs of the camera, supplying $\mathrm{HV}$ and LV and receiving the differential signals from the drivers in twisted pair cables. On the board a fuse for overcurrent protection of each LV line is provided. From the boards, round shielded cables carry the PMT signals on twisted pair wires to the front-end crate, located at the base of the camera support.

\section{Electronics and data acquisition system}

The FD telescopes are used to record fluorescence signals of widely varying intensity atop a sizeable, and continuously changing, light background. This presents a significant challenge for the design of the electronics and data acquisition system (DAQ), which must provide a large dynamic range and strong background rejection, while accepting any physically plausible air shower. The DAQ must also allow for the robust, low-cost, remote operation of the FD telescopes. And finally, the absolute FD-SD timing offset must be sufficiently accurate to enable reliable hybrid reconstruction.

The FD electronics are responsible for anti-alias filtering, digitizing, and storing signals from the PMTs. As the PMT data are processed, they are passed through a flexible three-stage trigger system implemented in firmware and software. The remaining high-quality shower candidates are packaged by an event builder for offline shower reconstruction. For each shower candidate, a hybrid trigger is generated for the surface detector. An overview of the complete trigger sequence is presented in Table 1 .

The organization of the electronics and DAQ is hierarchical, reflecting the physical layout of the FD buildings. Figure 16 shows the readout 


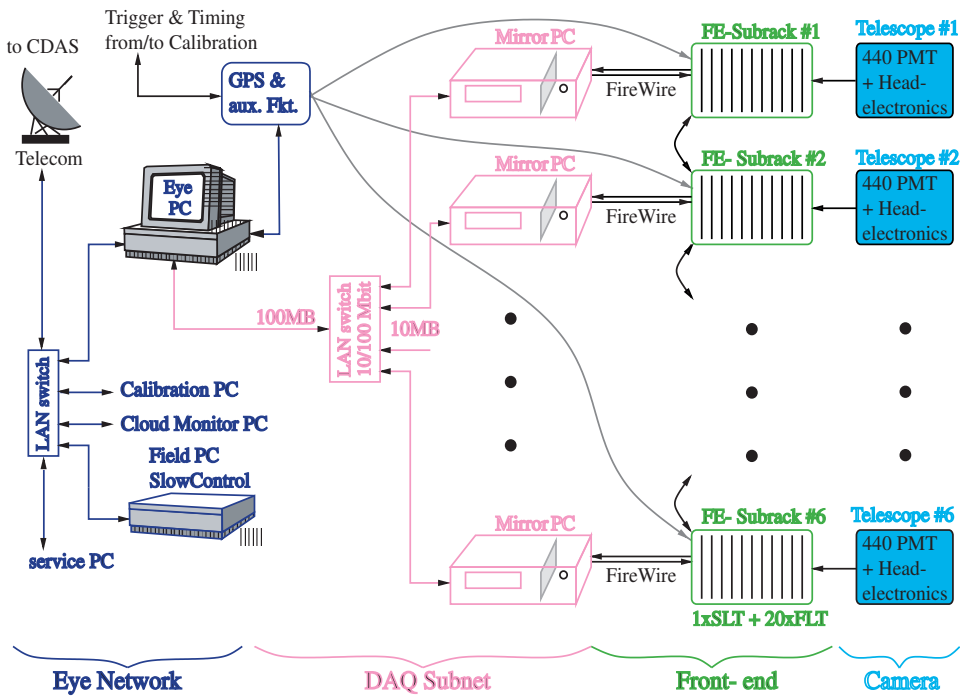

Figure 16: Readout scheme of an FD site The flow of recorded data is right to left.

scheme of one FD site, divided into four logical units: head electronics for 440 PMTs $\times 6$ telescopes, which provide low and high voltage; front-end (FE) sub-racks, where the signals are shaped and digitized, and where threshold and geometry triggers are generated; the DAQ subnet, in which six telescope PCs (MirrorPCs) read out the stored data and perform additional softwarebased background rejection; and an FD site network, in which a single EyePC merges triggers from the six telescopes and transfers them to the Observatory Central Data Acquisition System (CDAS) in Malargüe. The FD site network also contains a Slow Control PC to allow for remote operation of the building. The camera electronics and PCs are synchronized by a clock module based on the Motorola Oncore UT+ GPS receiver, the same receiver used in the SD array.

\subsection{Front-end electronics}

Each FD camera is read out by one front-end sub-rack and an associated MirrorPC. The front-end electronics contain 20 Analog Boards (ABs), and each $\mathrm{AB}$ receives data from a column of $22 \mathrm{PMT}$ channels. The boards are designed to handle the large dynamic range required for air fluorescence measurements; at the energies of interest for the Pierre Auger Observatory, this means a range of 15 bits and $100 \mathrm{~ns}$ timing 23]. The sub-racks also contain dedicated boards for hardware triggers: 20 First Level Trigger (FLT) boards for pixel triggers, and one Second Level Trigger (SLT) board for track identification within the camera image. 


\begin{tabular}{|c|c|c|c|}
\hline \multicolumn{4}{|c|}{ FD Trigger Sequence } \\
\hline Trigger Level & Location & Purpose & Event Rate \\
\hline $\begin{array}{c}1 \\
(\mathrm{FLT}) \\
\end{array}$ & $\begin{array}{l}\text { FE sub-racks } \\
\text { (FLT boards) }\end{array}$ & $\begin{array}{l}\text { pixel threshold } \\
\text { trigger }\end{array}$ & $100 \mathrm{~Hz}$ pixel $^{-1}$ \\
\hline $\begin{array}{c}2 \\
(\mathrm{SLT})\end{array}$ & $\begin{array}{c}\text { FE sub-racks } \\
\text { (SLT board) }\end{array}$ & $\begin{array}{l}\text { track shape } \\
\text { identification }\end{array}$ & $0.1-10 \mathrm{~Hz}$ telescope ${ }^{-1}$ \\
\hline $\begin{array}{c}3 \\
\text { (TLT) }\end{array}$ & $\begin{array}{l}\text { MirrorPCs } \\
\text { (software) }\end{array}$ & $\begin{array}{l}\text { lightning } \\
\text { rejection }\end{array}$ & $0.01 \mathrm{~Hz}$ telescope $\mathrm{e}^{-1}$ \\
\hline T3 & $\begin{array}{c}\text { EyePC } \\
\text { (software) }\end{array}$ & $\begin{array}{l}\text { event builder, } \\
\text { hybrid trigger }\end{array}$ & $0.02 \mathrm{~Hz}$ building ${ }^{-1}$ \\
\hline
\end{tabular}

Table 1: Trigger sequence for FD events. At each telescope, events are selected based on channel thresholds (FLT), track shape (SLT), and lightning rejection (TLT). Events passing the TLT are merged by an event builder on the FD EyePC. If the event passes further quality cuts for a simple reconstruction, a hybrid trigger (T3) is sent to CDAS.

\subsubsection{The Analog Board}

The purpose of the $\mathrm{AB}$ is to receive inputs from the head electronics on the PMT camera and adapt them for digitization by the analog-to-digital converters (ADCs) located on a corresponding FLT board. As shown in Fig.17, the analog and FLT boards are physically connected by three 50-pin SMC connectors. The combined front-end module measures $367 \mathrm{~mm} \times 220 \mathrm{~mm}$, and is housed in a $9 \mathrm{U}$ standard crate next to each FD telescope. Every crate contains 20 modules in total.

One $\mathrm{AB}$ channel comprises the following logical blocks: receiver, gain stage, anti-aliasing filter, and dynamic range adapter. The channel receives input from the HE on the PMT camera via a distribution board. Individual pixel enabling is performed by a fast analog switch, which is also used to generate an internal test pulse upon request from the FLT logic. In each channel, the AB:

- performs a differential to single-ended conversion of the signal;

- adjusts the channel gain;

- applies an anti-aliasing filter before signal sampling;

- adapts the 15-bit dynamic range to the 12-bit ADCs;

- provides an injection point for test pulses.

The telescope PMTs are coarsely gain-matched during the installation of the PMT camera to avoid expensive individual HV control. Additional fine-tuning of the channel gains is achieved by means of digital potentiometers connected in series with a resistor on the feedback loop of each non-inverting gain stage. The devices can change individual channel gains up to a factor of about 1.9, and allow for gain matching of the channels in the camera to within $0.6 \%$. 


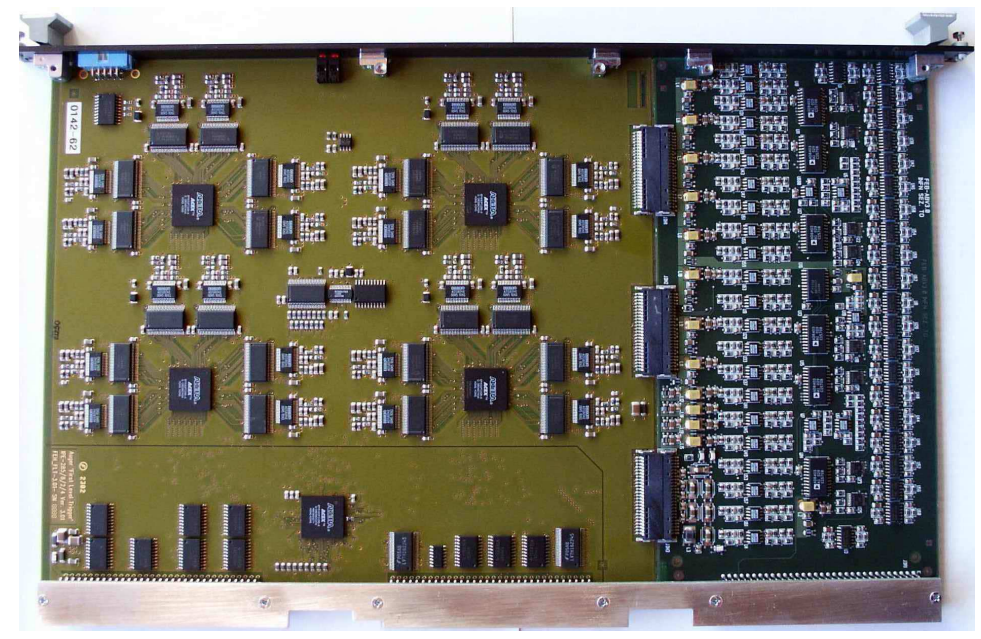

Figure 17: Photo of the FLT (left) and Analog Board (right): Both boards are connected by three 50-pin SMC connectors. A stiffener bar and common front panel provide mechanical rigidity.

Prior to sampling, the PMT signal is processed by an anti-aliasing filter to match the $10 \mathrm{MHz}$ digitization rate. A fourth-order Bessel filter with a cutoff frequency of $3.4 \mathrm{MHz}$ has been implemented in the $\mathrm{AB}$ as a compromise between reconstruction error and circuit complexity. The Bessel filter scheme, featuring a linear dependence of the transfer function phase with frequency, was selected after a detailed study of optimal filters to minimize distortion of the current signal shape.

The final component of an $\mathrm{AB}$ channel is the dynamic range adapter. The FD records signal sizes between 3 and $10^{5}$ photoelectrons per $100 \mathrm{~ns}$, covering a dynamic range of 15 bits. Rather than digitize the full range of signal sizes, an optimal cost-effective solution using dynamic range compression to 12-bit ADCs has been implemented in the analog electronics design.

The compression technique, which uses virtual channels, relies on the fact that the the shower signal does not appear on all pixels simultaneously; instead, each pixel is triggered in a well-ordered time sequence. Every channel is configured with a high and low gain of about 20 and 1, respectively. The high gain is optimized for the most frequent small- and intermediate-amplitude pulses, and is digitized pixel by pixel. The signal of the low gain stage is routed together with signals from 10 other non adjacent pixels in an analog summing stage. The sum signal is then processed by a virtual channel with a gain near unity.

When a nearby high-energy shower is detected by the FD, typically only one channel out of the group of 11 will saturate its 12-bit range at a given time. Even in case of multiple saturation in the same group, this will never occur in overlapping time bins. Therefore, the signal can be recovered from the virtual 


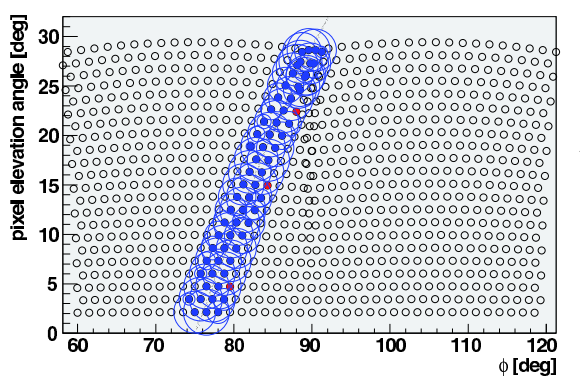

\section{Simulated shower at $\mathrm{d}=10 \mathrm{~km}$ \\ $\mathrm{E}=1.00 \mathrm{ZeV}$ \\ $X_{\max }=825 \mathrm{~g} / \mathrm{cm}^{2}$}
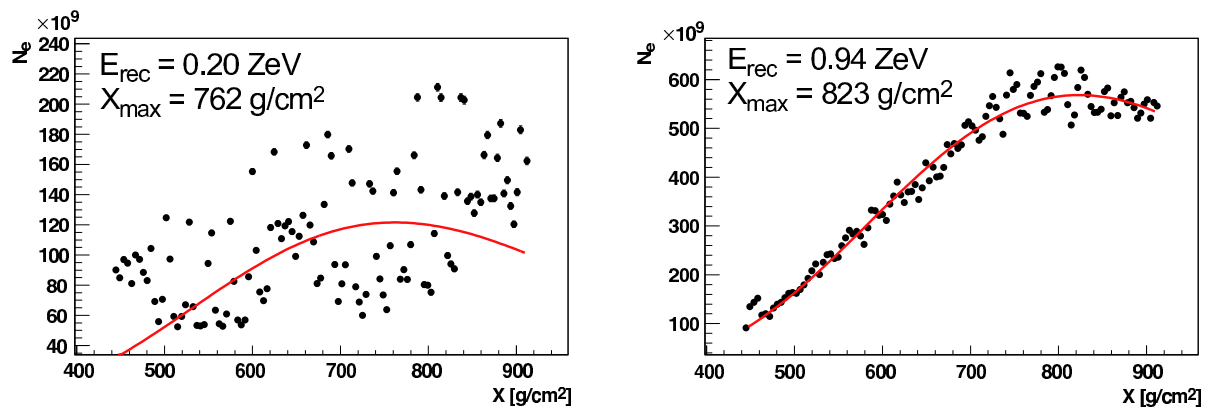

Figure 18: Simulated air shower of $10^{21} \mathrm{eV}$ at $10 \mathrm{~km}$ from the telescope. The top-left figure shows the light track in the cameras. The lower left figure shows the reconstructed shower profile without use of the virtual channel information. Making use of the virtual channel (lower right), the shower parameters can be reconstructed with minimal systematic distortions.

channel without ambiguity. This is demonstrated in Fig. 18 which depicts a simulated $10^{21} \mathrm{eV}$ shower with a landing point $10 \mathrm{~km}$ from the FD telescopes. The high-gain channels saturate during the development of the shower, and an attempt to reconstruct the shower using only these channels leads to significant systematic distortions in the shower profile. However, with the information in the virtual channel, the shower is reconstructed with almost no systematic biases [25].

\subsubsection{First Level Trigger (FLT) Module}

The First Level Trigger (FLT) module is the heart of the digital front-end electronics. The module processes the data from one 22-channel column. Its main tasks are to:

- continuously digitize signals from the $\mathrm{AB}$ at $10 \mathrm{MHz}$;

- store the digitized raw data in memory for later readout;

- measure the pixel trigger rate for each channel;

- compensate for changing background conditions and maintain a pixel trigger rate of $100 \mathrm{~Hz}$ by adjusting the pixel trigger thresholds; 
- allow access to raw data memory and internal registers;

- provide a digital interface to the $\mathrm{AB}$ to generate test pulses and to set the analog gain at the $\mathrm{AB}$;

- calculate the baseline offset and its fluctuation averaged over a $6.5 \mathrm{~ms}$ period;

- calculate the multiplicity (number of triggered pixels) in one column.

The functions of the FLT are implemented in FPGA (Field Programmable Gate Array) firmware to improve the flexibility and cost-effectiveness of the module. A pipelined 12-bit ADC (ADS804) is used to digitize the signal at $10 \mathrm{MHz}$ in each channel, and the data are stored with a 4-bit status word in $64 \mathrm{k} \times 16$-bit SRAMs. The address space of each SRAM is divided into 64 pages of 1000 words. In the absence of triggers from the SLT module (described in the next section), each page works as a circular buffer to hold the ADC values of the previous $100 \mu \mathrm{s}$. When an SLT trigger occurs, all FLT boards synchronously switch to the next unused memory page, whose address is provided by the SLT.

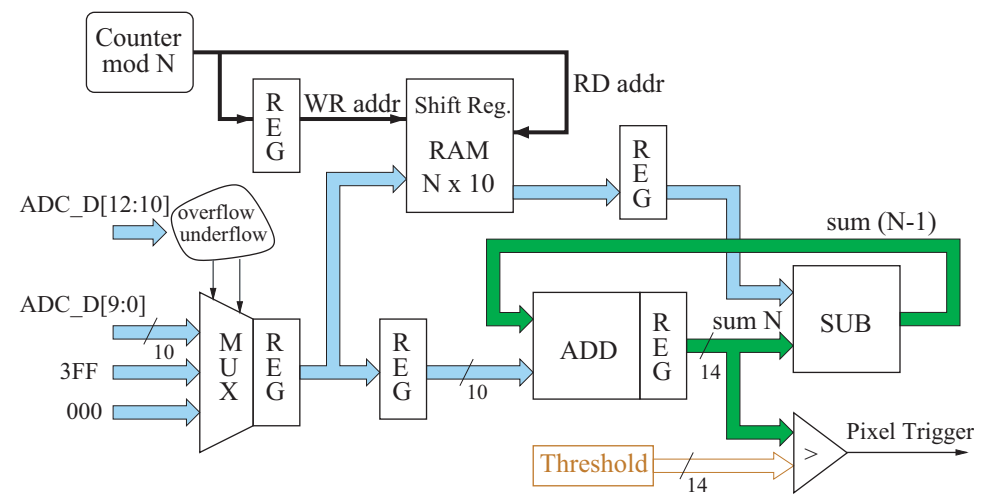

Figure 19: Generation of the First Level Trigger in one channel. A moving sum of 5 to 16 values is compared to an adjustable threshold. The use of the sum improves the S/N-ratio, and allows for regulation of the trigger rate.

The main task of the FPGA logic is to generate the pixel trigger (FLT) using a threshold cut on the integrated ADC signal. The FLT is shown schematically in Fig. 19, A moving "boxcar" sum of the last $n$ ADC samples is compared to a 14-bit threshold. Here $n$ is a fixed number of time bins which can be chosen in the range $5 \leq n \leq 16$. The threshold is dynamically adjusted to maintain a pixel trigger rate of $100 \mathrm{~Hz}$. When the sum exceeds the threshold, a pixel trigger is generated. The use of the sum substantially increases the signal-to-noise $(\mathrm{S} / \mathrm{N})$ ratio for each channel; for $n=16, \mathrm{~S} / \mathrm{N}$ improves by a factor of 2.8 [26]. When the moving sum drops below the threshold, a retriggerable mono-flop extends the pixel trigger for an adjustable period of $5 \mu$ s to $30 \mu$ s common to all pixels, increasing the chance of coincident pixel triggers. 
The rate of pixel triggers, called the "hit rate," is measured in parallel by counters for each channel. It is used to adjust the threshold in such a manner that the hit rate is kept constant at $100 \mathrm{~Hz}$ under variable background light conditions. The background light levels seen by each PMT can also be monitored by analysis of the variances of the ADC values. Therefore, the FPGAs regularly evaluate the mean and variance of the channel baselines every 6.5 ms using 65536 consecutive ADC samples [27]. The measured channel hit rates, thresholds, offsets, and variances are periodically queried and stored in a monitoring database for experimental control (see section 6.5).

Finally, the multiplicity, or the number of pixels triggered simultaneously within $100 \mathrm{~ns}$, is calculated for each FLT and for the full camera. The number of channels above threshold in one 22-pixel column is tracked on the FLT board. The total sum for the full camera is evaluated at the SLT board using daisychained lines on the backplane. The chronological sequence of multiplicity values carries information about the temporal development of the camera image, which is used by the software trigger (see section 4.2.1).

\subsubsection{The Second Level Trigger (SLT)}

The pixel triggers generated for each channel in the 20 FLT boards of the FE sub-rack are read out by a Second Level Trigger (SLT) board. The functions of the SLT are implemented in FPGA logic, and its primary task is to generate an internal trigger if the pattern of triggered pixels follows a straight track [28]. The algorithm searches for track segments at least five pixels in length. It uses the fundamental patterns shown in Fig. 20, as well as those created by rotations and mirror reflections of these segments.

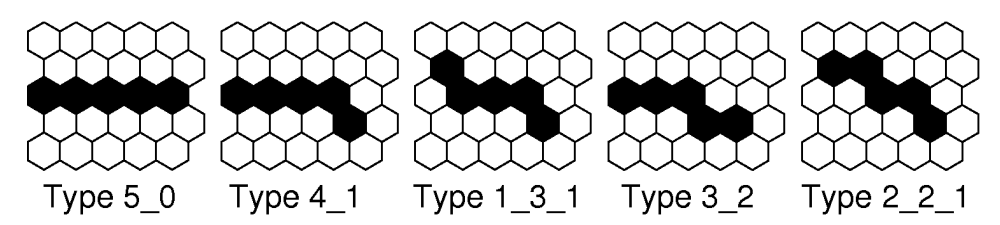

Figure 20: Fundamental types of pattern regarded as straight track segments.

During data acquisition, some tracks will not pass through every pixel center, and therefore some PMTs along the track may not record enough light to trigger. To allow for this situation, and to be fault-tolerant against defective PMTs, the algorithm requires only four triggered pixels out of five. Counting all different four-fold patterns originating from the five-pixel track segments in Fig. 20, one finds 108 different pattern classes.

A brute-force check of the full $22 \times 20$ camera pixel matrix for the 108 pattern classes would result in 37163 combinations in total. Therefore, the SLT uses a pipelined mechanism that scans for tracks on a smaller $22 \times 5$ sub-matrix of the camera. Every $100 \mathrm{~ns}$, the FLT pixel triggers of two adjacent columns $(22 \times 2$ 
pixels) are read into a pipelined memory structure. During the scan of the full camera, coincidence logic identifies the pattern classes 1 to 108 and generates a trigger if a pattern is found. The full scan lasts $1 \mu \mathrm{s}$, and the pixel trigger information scanned during this time is stored in the pixel memory of the SLT, which is organized as a ring buffer for 64 events (as on the FLT). The FLT multiplicities are also stored in the same memory, providing complementary measures of the spatial and temporal size of the event.

Note that in addition to generating the geometrical trigger, the SLT board also supplies bookkeeping information for the FE sub-rack. In particular, the board provides a time stamp for each event using synchronization signals sent by the GPS module over a dedicated line.

\subsubsection{Test Systems}

The large number of channels in each telescope and the complexity of the electronics and triggers require the use of an automatic testing system. The testing and calibration of the full optics-PMTs-electronics chain is described in section 5. However, the system also allows for tests of the electronics alone. A test-pattern generator has been implemented on the front-end boards to create pulses of variable width and amplitude at the input of each analog channel. These test pulses are used to check the analog and digital functions of the FLT and SLT boards. The system is independent of the full camera, and can be used for maintenance when the HV/LV supply of the camera is turned off.

\subsection{DAQ Software and Software Trigger}

Once an event has been processed by the DAQ hardware and stored in FLT and SLT memory, it can be read out and analyzed by trigger software in the MirrorPCs. Each MirrorPC is connected to the digital boards in the FE subrack via a FireWire interface 24], and the MirrorPCs communicate with the EyePC through a 10/100 Mbit LAN switch.

The DAQ system can handle data from three types of sources:

1. external triggers;

2. calibration events and test pulses;

3. real air shower events.

External triggers are primarily artificial light sources used for atmospheric monitoring, such as laser shots from the Central Laser Facility [9]. The various types of calibration pulses are described in section 5 .

Events triggered externally, or by the calibration and test-pulser systems, receive special trigger bits in the SLT status word to distinguish them from real air shower data. When the MirrorPCs read out such events from the FE modules, they are sent directly to the EyePC for event building and storage in dedicated raw data files. In contrast, when the MirrorPCs read out true air shower data, the events are processed by a Third Level Trigger. The surviving data are sent to the EyePC, which builds an event from the coincident data in all telescopes and generates a hybrid trigger (T3) for the surface array. 


\subsubsection{The Third Level Trigger (TLT)}

The Third Level Trigger (TLT) is a software algorithm designed to clean the air shower data stream of noise events that survive the low-level hardware triggers. It is optimized for the fast rejection of triggers caused by lightning, triggers caused by muon impacts on the camera (see Fig. 131), and randomly triggered pixels.

During the austral summer, distant lightning can significantly disrupt the normal data acquisition of the FD telescopes. In good atmospheric conditions, the SLT will detect one to two events per minute per telescope. However, lightning can cause large parts of the camera (i.e., hundreds of pixels) to trigger in bursts of several tens of events per second. If not filtered, the bursts will congest the 64-event buffers in the FLT and SLT boards, and the telescope readout (including ADC traces) will suffer a substantial increase in dead time.

The TLT is designed to efficiently filter lightning events without performing costly readouts of the complete ADC traces. The algorithm achieves high speed by reading out only the FLT multiplicities and the total number of triggered pixels. Using several thousand true showers and background events from one year of data, a dedicated study was performed to determine efficient lightning cuts based on the time development of the multiplicity and the integral of the multiplicity over the whole event consisting of 1000 subsequent multiplicity values [29]. With these cuts, approximately $99 \%$ of all lightning events are rejected by the TLT during a short $(50 \mu \mathrm{s})$ decision time.

The multiplicity-based lightning rejection effectively removes noise events with more than 25 pixels. The remaining events with smaller number of triggered pixels, which are contaminated by random pixel triggers and muon impacts, are filtered in a second step. The correlation between the spatial arrangement and peak signal times of triggered pixels is used to discard noisy channels far off the light track [29]. The full ADC traces must be used to determine the pixel trigger times, but this readout does not appreciably increase the telescope dead time when the number of pixels is small.

The TLT performance has been validated with simulated showers and data recorded during different weather and sky background conditions. Although the exact behavior of the algorithm depends on the actual conditions, approximately $94 \%$ of all background events are correctly identified by the TLT. The fraction of true showers rejected by the trigger is below $0.7 \%$.

\subsubsection{Hybrid Trigger (T3)}

Events passing the TLT in each telescope are sent to the EyePC through the DAQ subnet. An event builder running on the EyePC merges coincident events from adjacent telescopes. The EyePC also sends a hybrid trigger, called a T3, to the CDAS in Malargüe.

The T3 acts as an external trigger for the surface array. Its purpose is to record hybrid events at low energies (below $3 \times 10^{18} \mathrm{eV}$ ) where the array is not fully efficient and would not often generate an independent trigger. Hybrid events at these energies occur within $20 \mathrm{~km}$ of the FD buildings and usually do 
not trigger more than one or two SD stations. However, as discussed in section 7 even limited SD data are sufficient for high-quality hybrid reconstruction.

The T3 algorithm is used to calculate a preliminary shower direction and ground impact time with a simple online reconstruction. Once these data arrive at the CDAS, a request is sent to the SD for signals recorded close to the calculated impact time. For each T3 trigger, the SD stations nearest the FD building (comprising approximately one-quarter of the array) are read out. The FD and SD data are merged offline for subsequent hybrid analysis.

\section{Calibration}

\subsection{Introduction}

The reconstruction of air shower longitudinal profiles and the ability to determine the total energy of a reconstructed shower depend on being able to convert ADC counts to a light flux at the telescope aperture for each channel that receives a portion of the signal from a shower. It is therefore necessary to have some method for evaluating the response of each pixel to a given flux of incident photons from the solid angle covered by that pixel, including the effects of aperture projection, optical filter transmittance, reflection at optical surfaces, mirror reflectivity, pixel light collection efficiency and area, cathode quantum efficiency, PMT gain, pre-amp and amplifier gains, and digital conversion. While this response could be assembled from independently measured quantities for each of these effects, an alternative method in which the cumulative effect is measured in a single end-to-end calibration is employed here.

The absolute calibration of the fluorescence detectors uses a calibrated 2.5 m diameter light source (known as the "drum") at the telescope aperture, providing same flux of light to each pixel. The known flux from the light source and the response of the acquisition system give the required calibration for each pixel. In the lab, light source uniformity is studied using CCD images and the intensity is measured relative to NIST-calibrated photodiodes [33]. Use of the drum for gain adjustment and calibration provides a known, uniform response for each pixel in each camera of the FD detector. The average response of the FD is approximately 5 photons/ADC bin.

Three additional calibration tools are used as well:

- Before and after each night of data taking a relative calibration of the PMTs is performed. This relative calibration is used to track both short and long term changes in detector response.

- The relative FD response has been measured at wavelengths of 320, 337, 355, 380 and $405 \mathrm{~nm}$, defining a spectral response curve which has been normalized to the absolute calibration.

- An independent check of the calibration in some phototubes is performed using vertical shots from a portable laser in the field.

The sections below describe the hardware and use of these calibration systems. 


\subsection{Light source and drum}

The technique [30] is based on a portable $2.5 \mathrm{~m}$ diameter, $1.4 \mathrm{~m}$ deep, drumshaped light source which mounts on the exterior of the FD apertures (see Fig. 21). The source provides a pulsed photon flux of known intensity and uniformity across the aperture, and simultaneously triggers all the pixels in the camera. The surface of the drum is a good Lambertian. This means that the light emitted per unit solid angle from any small area $A$ depends only on the angle $\theta$ with respect to the normal direction according to $I(\theta)=I_{0} A \cos (\theta)$. Looking at the disk from angle $\theta$ and distance $d$ with a fixed solid angle $\omega$, the viewed disk area is $d \omega / \cos (\theta)$. So the intensity is $I(\theta)=I_{0} d \omega$. The intensity is independent of viewing angle for a perfect Lambertian. Fig. 22] shows that pixels of a CCD camera (each seeing a small portion of the drum surface) measure the same intensity regardless of the viewing angle to the drum.

To produce diffuse light inside the drum, illumination is provided by a pulsed UV LED [31] $(375 \pm 12 \mathrm{~nm})$, mounted against the face of a $2.5 \mathrm{~cm}$ diameter $\times 2.5 \mathrm{~cm}$ long Teflon ${ }^{\mathrm{TM}}$ cylinder. The Teflon cylinder is mounted in a $15 \mathrm{~cm}$ diameter reflector cup, which is mounted flush to the center of the drum front surface, illuminating the interior and the back surface of the drum. The LED is inserted down the axis of the drum from the back through a pipe. A silicon detector attached to the opposite end of the teflon cylinder monitors the light output for each pulse of the LED.

The drum was constructed in sections, using laminations of honeycomb core and aluminum sheet. The sides and back surfaces of the drum interior are lined with Tyvek ${ }^{\mathrm{TM}}$, a material diffusively reflective in the UV. The reflecting surfaces of the cup are also lined with Tyvek. The front face of the drum is a $0.38 \mathrm{~mm}$

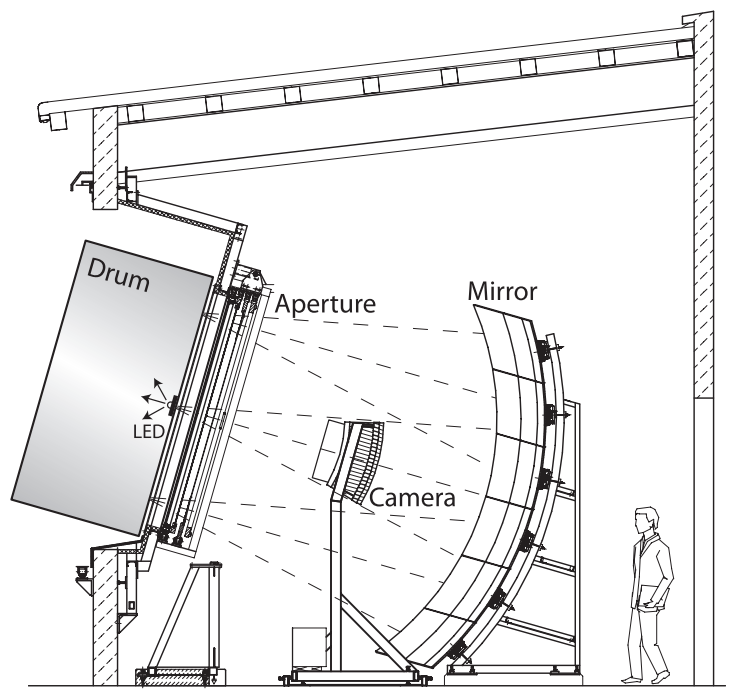

Figure 21: A schematic showing the drum mounted in a telescope aperture. 
thick Teflon sheet, which transmits light diffusively.

\subsection{Calibration of the drum}

The absolute calibration of the drum light source intensity is based on UVenhanced silicon photodetectors, calibrated at NIST to $\pm 1.5 \%$. While the small surface area and low response of these detectors preclude detection of the small photon flux from the drum surface directly, the photodiode calibration can be transferred to a more sensitive PMT/DAQ system.

To establish the absolute flux of photons emitted from the drum surface, a reference PMT is placed on the drum axis, $14 \mathrm{~m}$ from the surface. The LED light source in the drum is pulsed for a series of $5 \mu$ s pulses and the charge from the PMT is integrated and recorded for each pulse, resulting in a histogram of the distribution of the observed integrated flux.

On an optical bench, the PMT is then exposed to a small diffuse LED light source with neutral density filter. This filter adjusts the light intensity to the level similar to that of the drum. The LEDs are pulsed with the same driving circuitry as for the drum, and the intensity is set to a series of values producing a series of histograms with PMT centroids surrounding that from the drum measurement above. At each of these intensity settings a second measurement is made in which the PMT is replaced by the NIST-calibrated photodiode and a neutral density filter in the source is removed, increasing the intensity to a level measurable by the photodiode. For this second measurement, the LEDs are run in DC mode. The relationship of PMT response to photodiode current is found to be very linear. The flux of photons at $14 \mathrm{~m}$ from the drum surface is calculable from the active area of the photodiode, the neutral density filter reduction factor, the LED pulsed/DC duty factor, the NIST calibration for the photodiode, and the value of photodiode current corresponding to the PMTdrum centroid. The value of photodiode current is interpolated from the linear response-current relationship given above. The resulting uncertainty in the drum intensity is $6 \%$. Additional uncertainty contributions related to use of the drum at the FDs [35], such as temperature dependencies, along with camera response variations in time and spectral characteristics of the LED light source, combine with the drum intensity uncertainty resulting in an overall uncertainty of $9 \%$ for the absolute FD calibration.

\subsection{Drum relative uniformity measurements}

Uniformity of light emission from the drum surface is important, since the pixels in a FD camera view the aperture at varying angles. In addition, for each pixel, a different part of the aperture is blocked by the camera itself. Studies were made of uniformity of emission across the surface and as a function of viewing angle. These uniformity measurements were made using a CCD, viewing the emitting surface of the drum from a distance of $14 \mathrm{~m}$ (see Fig. 22). Images were recorded with the drum axis at angles of $0,10,20$ and 25 degrees relative to the CCD axis, covering the range of the Auger telescope field of view $\left(0-21^{\circ}\right)$. For these images, the UV LEDs were powered continuously. 
A $0^{\circ}$ image was used to analyze the uniformity of emission over the drum surface. Using software, concentric circles were drawn on the image, defining annular regions of increasing radius, as shown in the figure. The intensity of the pixels in each region was analyzed to obtain the intensity as a function of radius. In the area defined by the $2.2 \mathrm{~m}$ aperture radius, the relative uniformity of intensity is constant over the area to about $\pm 2 \%$ except for a central dark spot. The variation with viewing angle of a section of the drum image is also shown in Fig. 22 .
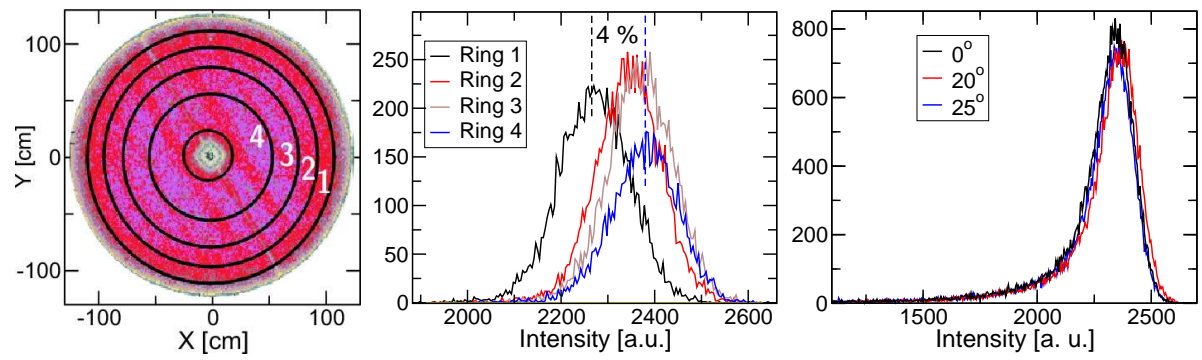

Figure 22: Left: $\mathrm{CCD}$ image at $0^{\circ}$ drum angle, showing the defined rings for relative intensity analysis. Production deformations in the teflon material can be seen. Middle: A plot of the observed pixel intensities in the defined regions of the drum, shown in the left panel; Right: The results of angular relative intensity measurements at 0,20 , and $25^{\circ}$. inclinations of the drum with respect to the CCD camera.

The measured drum non-uniformities are small (diagonal stripes in Fig. 22 intensity decrease with radius, etc.), indicating that the FD pixels see similar intensities integrated over the drum surface. While perfect drum uniformity is desirable, the present non-uniformities are acceptably small and well mapped over the surface of the drum. A ray-tracing program using the uniformity and angular intensity information from the CCD images shows less than $1 \%$ variation in total flux seen by the pixels, and corrections are applied for these variations.

\subsection{Absolute calibration using vertical laser shots}

The drum technique for absolute calibration has been checked for some pixels using remote laser shots at 337 and $355 \mathrm{~nm}$ 34, 35]. A laser pulse is shot vertically into the air with a known intensity. A calculable fraction of photons is scattered to the aperture of the FD detector. This yields a known number of photons arriving to the detector for each pixel, which views a segment of the laser beam. The response of each pixel to the known number of photons constitutes an absolute end-to-end calibration for those pixels. A calibrated laser probe is used to measure the number of photons in typical laser pulses in the field calibration. A nitrogen laser $(337 \mathrm{~nm})$ at a distance of $4 \mathrm{~km}$ is well suited for such purposes, because the scattered light flux is in the correct range and uncertainties due to aerosols are minimal. The scattering angles are just greater than $90^{\circ}$, where the differential scattering cross section is minimized for aerosols. Moreover, at a distance of $4 \mathrm{~km}$, the extra scattering from the 
beam by aerosols is approximately canceled by the extra aerosol attenuation of the primarily Rayleigh-scattered light flux. Therefore, the roving laser provides an independent and redundant absolute calibration of the FD cameras, based on the measured light injected into the atmosphere. Using the recorded laserinduced $\mathrm{ADC}$ traces and the drum calibration constants, we can then compare the number of predicted to measured photons.

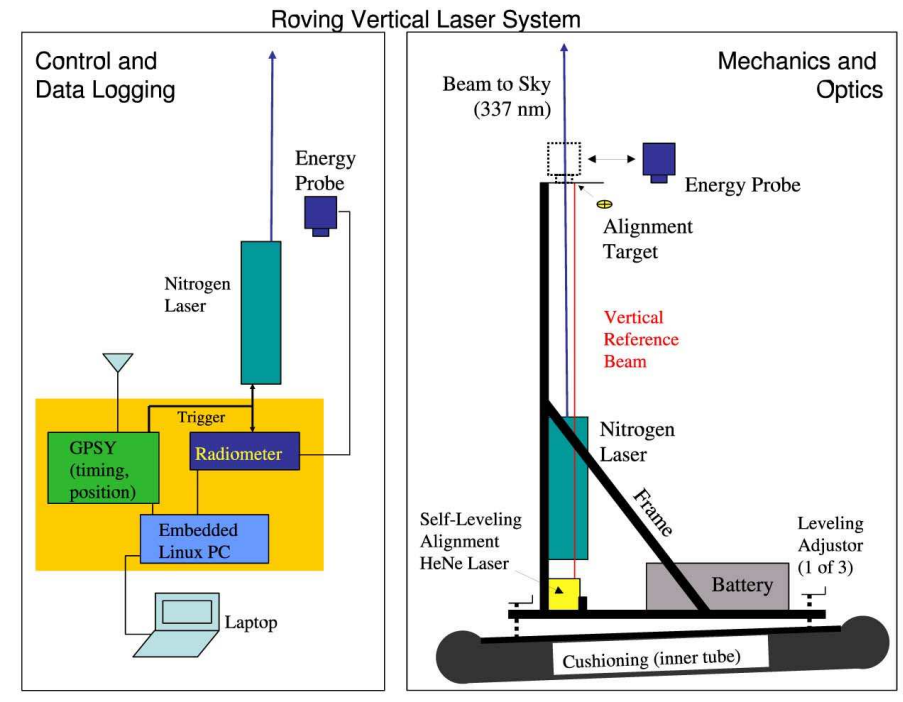

Figure 23: Roving vertical nitrogen laser hardware and control system.

Schematics of the hardware and controlling electronics for the roving laser system are shown in Fig. 23. The roving laser system presently in use is based on a $337 \mathrm{~nm}$ nitrogen laser, providing about $100 \mu \mathrm{J}$ per pulse, while the output is inherently unpolarized. The RMS values of 100 shots in a set, fired over 200 seconds, is typically $3 \%$. Variations of average energy in a 100 shot group is $2 \%$ on the time scale of 15 minutes, and $10 \%$ from night to night, as measured with the energy probe inserted into the beam for 100 shots before and after the calibration shots. A self-leveling He-Ne laser provides a reference beam within $0.03^{\circ}$ of vertical, allowing alignment of the nitrogen laser to within $0.1^{\circ}$ of vertical.

An embedded Linux PC, communicating via serial connection to a programmable GPS timing module, controls the laser firing time relative to the GPS second. The time stamp is used to identify calibration events for reconstruction.

For comparison with the $375 \mathrm{~nm}$ drum calibration, the measured relative FD response at the two calibration wavelengths must be known. The relative response has been measured, and is described in the next section.

Overall uncertainties of the roving laser calibration have been assessed at $12 \%$, dominated by laser probe calibration and atmospheric effects. 


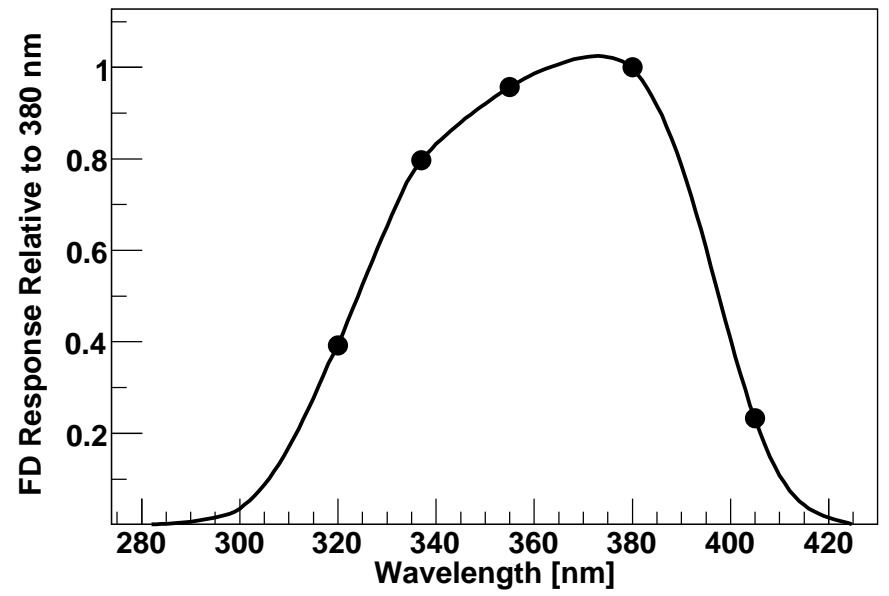

Figure 24: The results of the multi-wavelength measurements (see text, and [37].)

\subsection{Multi-wavelength calibration}

For calibration at wavelengths spanning the FD acceptance, a xenon flasher is mounted at the back of the drum, with a filter wheel containing 5 notch filters for selection of wavelengths. The xenon flasher [36] provides $0.4 \mathrm{~mJ}$ optical output per pulse covering a broad UV spectrum, in a time period of a few hundred nanoseconds. A focusing lens at the filter wheel output maximizes the intensity through the filter wheel and into the light pipe.

Relative drum intensity measurements at wavelengths of $320,337,355,380$ and $405 \mathrm{~nm}$ have been made with the same reference PMT used in the absolute measurements. At each wavelength the recorded response from the reference PMT, combined with the PMT quantum efficiency and corrected for light source and filter width effects, yield a quantity that is proportional to the number of photons emitted from the drum. The FD response detected using the various filters, with the drum placed in the aperture, can be combined with the results from the laboratory to form the curve of relative camera response [37] shown in figure 24

The curve in figure 24 is the result of an iterated spline fit beginning with a response curve predicted from manufacturer's specifications for FD components. The shape of this initial curve is dominated by the FD PMT QE and the UV filter transmission. The final fit includes effects of the notch filter transmission widths (15 nm FWHM), the reference PMT QE, the xenon light source emission spectrum, and the relative drum intensity for each filter, all measured in the lab, and the observed FD response to the drum for each filter. The relative uncertainty at each wavelength on the curve is $5 \%$. 


\subsection{FD relative calibration system}

The relative optical calibration system [35] is used to monitor detector response and to track absolute calibration between drum calibrations. The system is used before and after each night of operation. Three positions (A, B, and C) are illuminated for each camera, monitoring different groups of detector components. Light is distributed through optical fibers, from permanently installed light sources. All components use quartz optics.

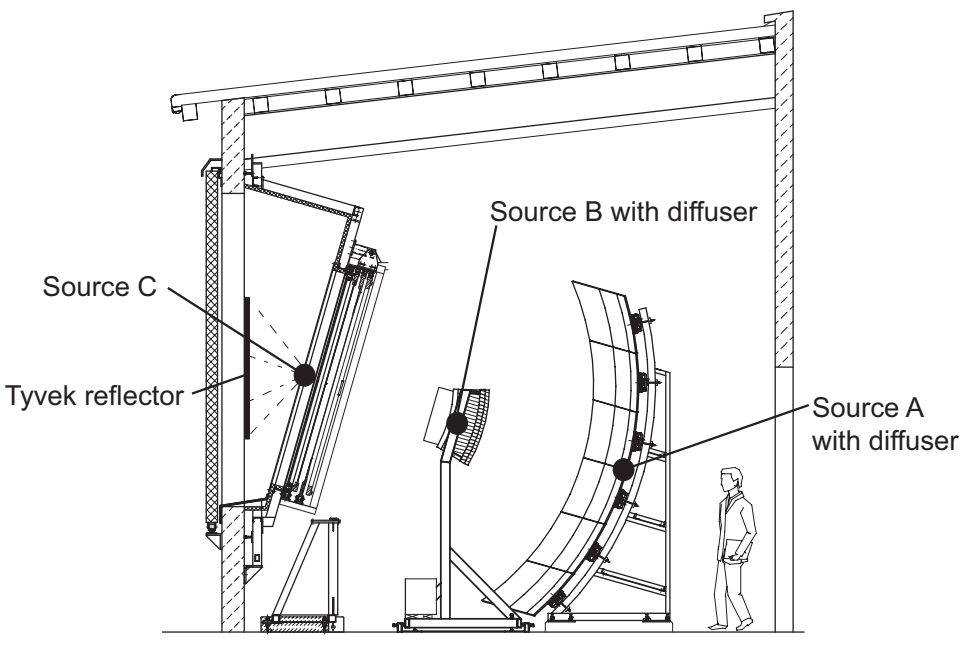

Figure 25: A schematic showing positions of light sources for three different relative calibrations of the telescope.

The A fiber light source is a $470 \mathrm{~nm}$ LED [32]. A 7:1 splitter at the source provides light to 6 fibers, running to a $1 \mathrm{~mm}$ thick Teflon diffusor located at the center of each mirror in the FD building, illuminating the camera face. The remaining fiber provides light for an output monitoring photodiode at the source. The A fiber LED is driven by a constant current source circuit. Normal operation pulses the LED for a series of $60 \mu$ s pulses.

Each of the B and C light sources are xenon flash lamps [36]. Each source is mounted at the focus of a $\mathrm{f} / 1.5$ lens, with downstream optics including a beam splitter (for a source monitoring fiber), a filter wheel, and an f/2.4 lens focusing onto a 7:1 splitter. The 7 fibers run to each fluorescence telescope, and to a monitor for each output, as for the A fiber. The B source fibers are split near each camera and terminate at $1 \mathrm{~mm}$ thick Teflon diffusors located at the sides of the camera, with the light directed at the mirror. The $\mathrm{C}$ source fibers are also split, and terminate outside the aperture with the light directed outwards. Tyvek sheets are mounted on the inside of the aperture shutters. The sheets are positioned such that they are opposite the fiber ends when the shutters are closed, and the diffuse light scattered off the Tyvek enters the aperture.

The B source includes a Johnson-U filter, approximating the full wavelength 
acceptance of the fluorescence telescopes. The $\mathrm{C}$ source filter wheel containing interference filters is centered at wavelengths of 330, 350, 370, 390 and $410 \mathrm{~nm}$, for monitoring detector stability at wavelengths spanning the spectral acceptance.

\section{Performance, operation and monitoring of the detector}

All four fluorescence sites have been completed and are in operation. Los Leones has been in full operation since March 2004 and Coihueco since July 2004. Los Morados began data acquisition in April 2005, and the fourth site at Loma Amarilla started its operation in February 2007.

\subsection{Uptime Fraction}

The operation of the fluorescence detector can be characterized by the uptime, or the fraction of the total time in which the FD was acquiring data. Anything that disables the measurement is considered as dead time. The main contributions to the dead time are the presence of the sun and nearly full moon on the sky, poor weather and the presence of the moon in any phase within $5^{\circ}$ in the FOV of a telescope. The position of the moon can be calculated in advance and the shutters of individual telescopes are closed when the moon approaches.

The shutters are also automatically closed when the weather conditions become dangerous for operation (high wind speed, rain, snow, etc.) and when the observed sky brightness (caused mainly by scattered moonlight) is too high. The influence of weather effects depends on the season, with the worst conditions typically occurring during Argentinian summer. Other periods of dead time are caused by the activity of atmosphere monitoring instruments, mostly by lidar stations and the Central Laser Facility, readout of the electronics, and any hardware or software problems.

The value of uptime fraction has been derived from the data and is crosschecked by several techniques. The average uptime fraction for the whole observation period is around $13 \%$ of the total time since operations commenced. Averaged uptime fractions for individual telescopes are shwon in Fig. 26. For Los Leones and Coihueco the values refer to the period January 2005 to January 2008, for Los Morados and Loma Amarilla the average is calculated from the individual start of operation till January 2008.

The Loma Amarilla building does not yet have a dedicated power line. The site is powered by a generator, which is less reliable and has caused a lower uptime compared to other FD sites.

\subsection{Background conditions}

The presence of the moon above the horizon increases the background light level, which has to be monitored. The direct current induced by the background is eliminated by the AC coupling of the PMT base, but it is possible to determine background levels using the direct relation between the fluctuations of the sky background and the photon flux. The analysis of the fluctuations in the ADC 


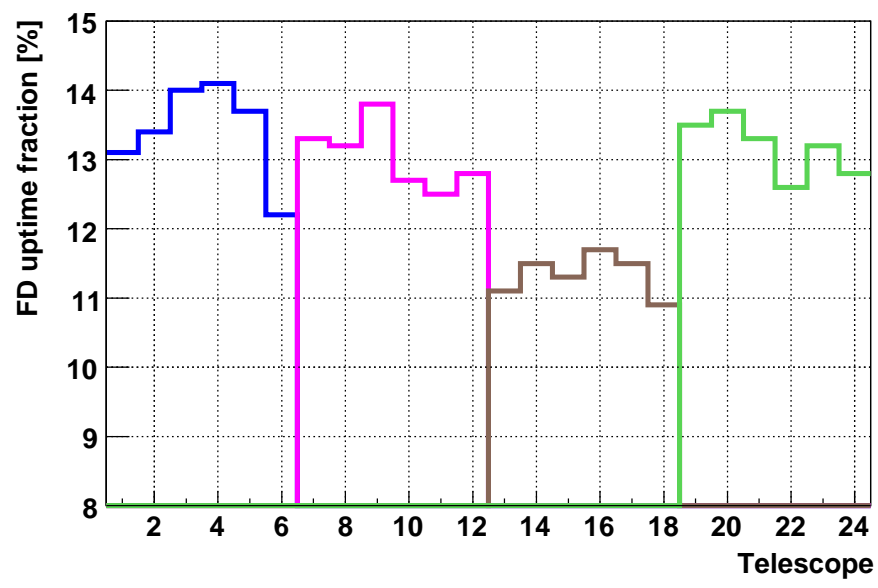

Figure 26: Uptime fraction for fluorescence telescopes. Telescopes are numbered as follows: Los Leones (south) site 1-6, Los Morados (east) site 7-12, Loma Amarilla (north) site 13-18, Coihueco (west) site 19-24.

signal (variance analysis) performed for each night of data taking is used to monitor the FD background signal and data taking conditions. Thus the amount of light is derived from ADC variance value and it is given in units (ADC counts) $)^{2}$.

The total background signal is the sum of the electronics background (photomultiplier and electronics noise) and the sky brightness (airglow, moonlight, stars and planet light, zodiacal light, twilight and artificial light) [27]. The typical values of background signals are: $3-5$ (ADC counts) ${ }^{2}$ for electronics background, around 20 (ADC counts) ${ }^{2}$ for cloudy nights, and between 25 and

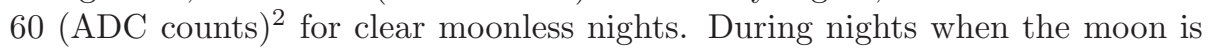
above the horizon, the light background level can reach several hundred (ADC counts) $)^{2}$.

The optimal background conditions for observation range from 25 to 60 (ADC counts) ${ }^{2}$ which corresponds to photon background flux from approximately 100 to 250 photons $\mathrm{m}^{-2} \mathrm{deg}^{-2} \mu \mathrm{s}^{-1}$. Under such conditions, one event per 2 hours is recorded for which the energy is determined to within $20 \%$ and the depth of maximum measured with an uncertainty of better than $40 \mathrm{~g} / \mathrm{cm}^{2}$. These high-quality events are the ones used for physics analysis.

\subsection{Standard Operation}

The FD operation is not fully automated and at present the assistance of a shift crew of at least two people per night is necessary. Their responsibilities consist of several activities before, during and after measurement each night. These include relative calibration of the cameras and optical components before and after observation, starting and stopping data taking according to 
weather conditions, prompt correction of software or hardware defects, etc. The operation of the FD is still evolving, and the software development is steadily transferring the responsibility of the human crew to automatic operation. The ultimate aim is to operate FD telescopes in fully automatic and remotely controlled mode from abroad.

FD is operated in nights with moon fraction below $60 \%$ beginning at the end of the astronomical twilight till the beginning of the next astronomical twilight. These criteria have evolved over time; before January 2005 the maximal illuminated moon fraction was $50 \%$, and hence data taking period was 2 days shorter per month. The observation period lasts 16 days per month, with an average observational time of about 10 hours (ranging from about 14 hours in June to 5 hours in December).

\subsection{Slow Control System}

The fluorescence detectors are operated from the central campus in Malargüe, and are not operated directly from the FD buildings. Therefore, the main task of the Slow Control System (SCS) is to ensure a secure remote operation of the FD system. The SCS works autonomously and continuously monitors detector and weather conditions. Commands from the remote operator are accepted only if they do not violate safety rules that depend on the actual experimental conditions: high-voltage, wind speed, rain, light levels inside/outside the buildings, etc. In case of external problems such as power failures or communication breakdowns the SCS performs an orderly shutdown, and also a subsequent startup of the fluorescence detector system if the conditions have changed. If parts of the SCS itself fail, the system automatically reverts to a secure mode as all potentially critical system states (open shutters, high-voltage on, etc.) have to be actively maintained.

To ensure reliable supervision and to allow for high flexibility and stability, the SCS is based on industrial PROFIBUS components. This bus system consists of several bus terminals with specific functions, such as analog input, digital output, relays, etc. The terminals make up a modular bus-system which is addressed and controlled from a PC. The slow control PC is the central instance of the SCS in each FD building and runs the main control software under a Windows operating system. The system for one of the fluorescence detector buildings is sketched in Fig. 27.

The shift crew interacts with the control-system via web-browsers. One central webserver communicates via OPC-XML gateways with 4Control OPCservers on the control-PCs. Several views with different levels of detail display the status, from an overview of the whole fluorescence detector of the Observatory down to single telescopes (see Fig. 28).

For the further automation of the data taking, an interface between the Slow Control and the Data Acquisition systems is under development. The final goal

is an automatic, scheduled operation where the shifters have to react only to malfunctions of the system. 


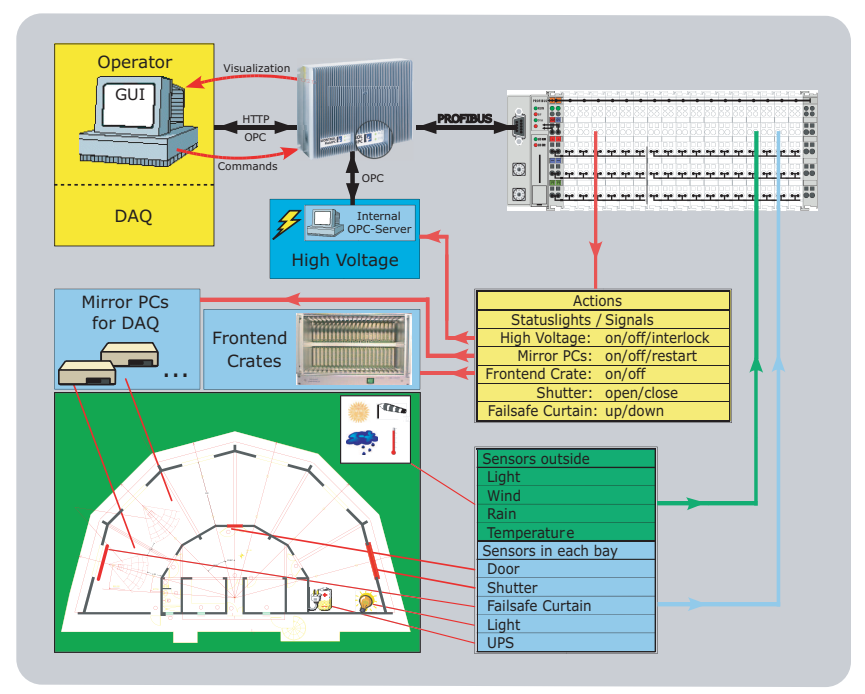

Figure 27: Schematic view of the slow-control system

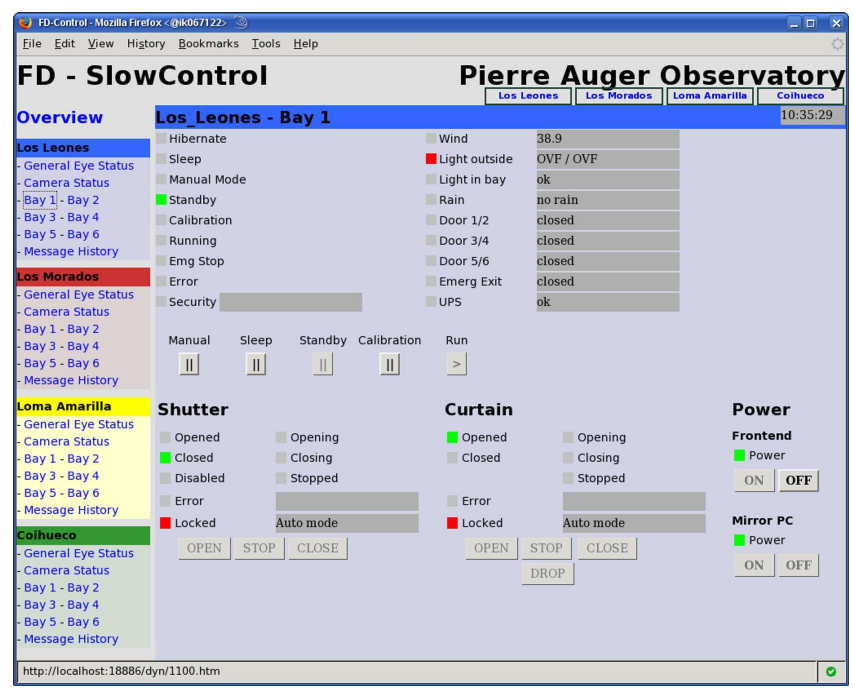

Figure 28: SCS display of a FD telescope

\subsection{Monitoring}

FD data-taking can only take place under specific environmental conditions. The light-sensitive cameras must be operated on dark nights with low wind and without rain. This makes the operation a full task for the shifters, who judge the suitability of operations on the basis of the information given by the SCS. 
The telescope performance must be monitored constantly to assure the quality of the recorded data, as well as guarantee the safe operation of all detector components. A user-friendly monitoring tool has been developed to support the shifters in judging and supervising the status of the detector components, the electronics, and the data-acquisition.

The monitoring tool [38] is built upon online MySQL databases, organized by FD building. The databases record data from regular FD measurements and from calibrations and atmospheric surveys. The data are transferred to a central server at the Malargüe campus using the internal MySQL database replication mechanism. This mechanism recognizes communication problems and tries to synchronize database changes when the connection is reestablished; this guarantees the completeness of the dataset on the central server, even if the information are not available online immediately due to network failures. Fig. 29 shows the schematic layout of the databases.

The user interface is based on a webserver running Apache. The website uses PHP, CSS and JavaScript; graphs and custom visualizations are implemented using the JPGraph package for direct PHP calls to the database. They are dynamically generated to be accessible not only for the shifter, but also remotely for experts. Graph explanations as well as troubleshooting tips are available through linked wiki pages. Alarms are triggered automatically in case of special occurrences. This tool helps the shifter to monitor constantly the performance of the detector to assure the quality of the recorded data as well as guaranteeing the safe operation of all detector components. In addition it offers a unique opportunity to monitor the long term stability of some key quantities and the data quality.

\subsection{Online events}

Besides using the online monitoring system for a continuous performance check by integrating over the most recent history of data, the operators are furnished with an online event display of the cameras. Two typical event examples corresponding to a real cosmic ray air shower and to a background

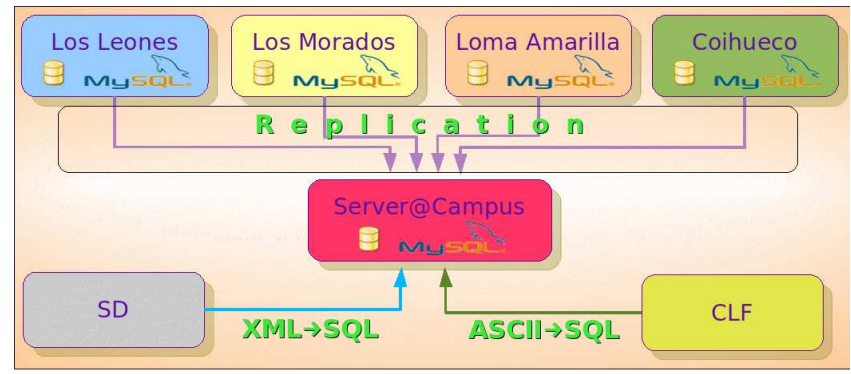

Figure 29: Organisation of the databases: The single databases at each FD-building are replicated to the database server at the central campus, while other sources like the SD insert data directly into the central database. 


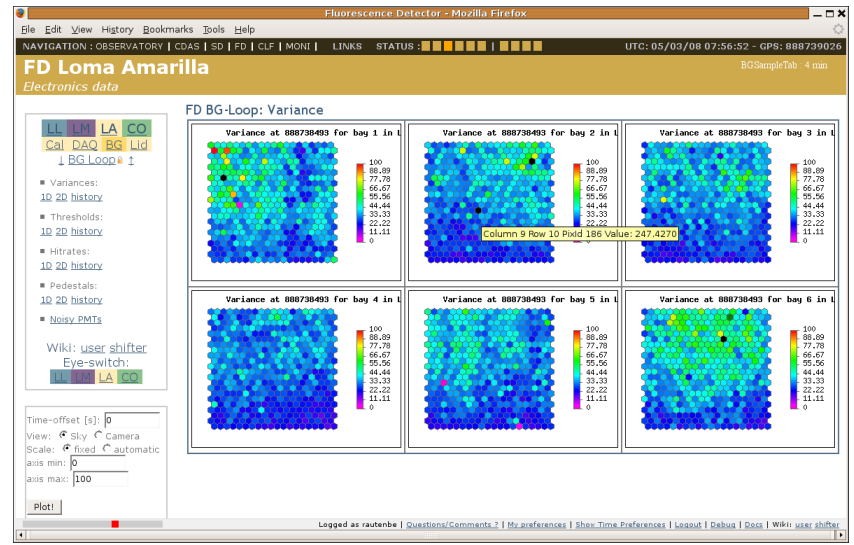

Figure 30: Screenshot of the FD monitoring web interface, showing a selection of FD background data for all pixels in the six cameras at Loma Amarilla.

event are shown in Fig. 31. The light spot of the air shower, shown in the upper part of the figure, takes about $10 \mu \mathrm{s}$ to cross the FOV of the camera while individual pixels are illuminated for up to about $1 \mu \mathrm{s}$. This is clearly different from the event shown in the bottom part of the figure. Here, all activated pixels show huge narrow pulses all starting in the same sample of the $10 \mathrm{MHz}$ ADC. Such a geometrical pixel pattern and timing feature is incompatible with an air shower and is most likely due to a muon penetrating the camera.

\section{Event Reconstruction}

\subsection{Geometrical Reconstruction}

A hybrid detector achieves the best geometrical accuracy by using timing information from all the detector components, both FD pixels and SD stations. Each element records a pulse of light from which it is possible to determine the time of the pulse and its uncertainty. Each trial geometry for the shower axis yields a prediction for the signal arrival times at each detector component.

Differences between actual and predicted times are weighted using their corresponding uncertainties, squared, and summed to construct a $\chi^{2}$ value. The hypothesis with the minimum value of $\chi^{2}$ is the reconstructed shower axis.

In the $\mathrm{FD}$, cosmic ray showers are detected as a sequence of triggered pixels in the camera. An example of an event propagating through two adjacent FD telescopes is presented in Fig. 32 The first step in the analysis is the determination of the shower-detector plane (SDP). The SDP is the plane that includes the location of the eye and the line of the shower axis. (See the sketch in Fig. 33) Experimentally, it is the plane through the eye which most nearly contains the pointing directions of the FD pixels centered on the shower axis. (See fitted line in Fig. 32) Using a known axis provided from 


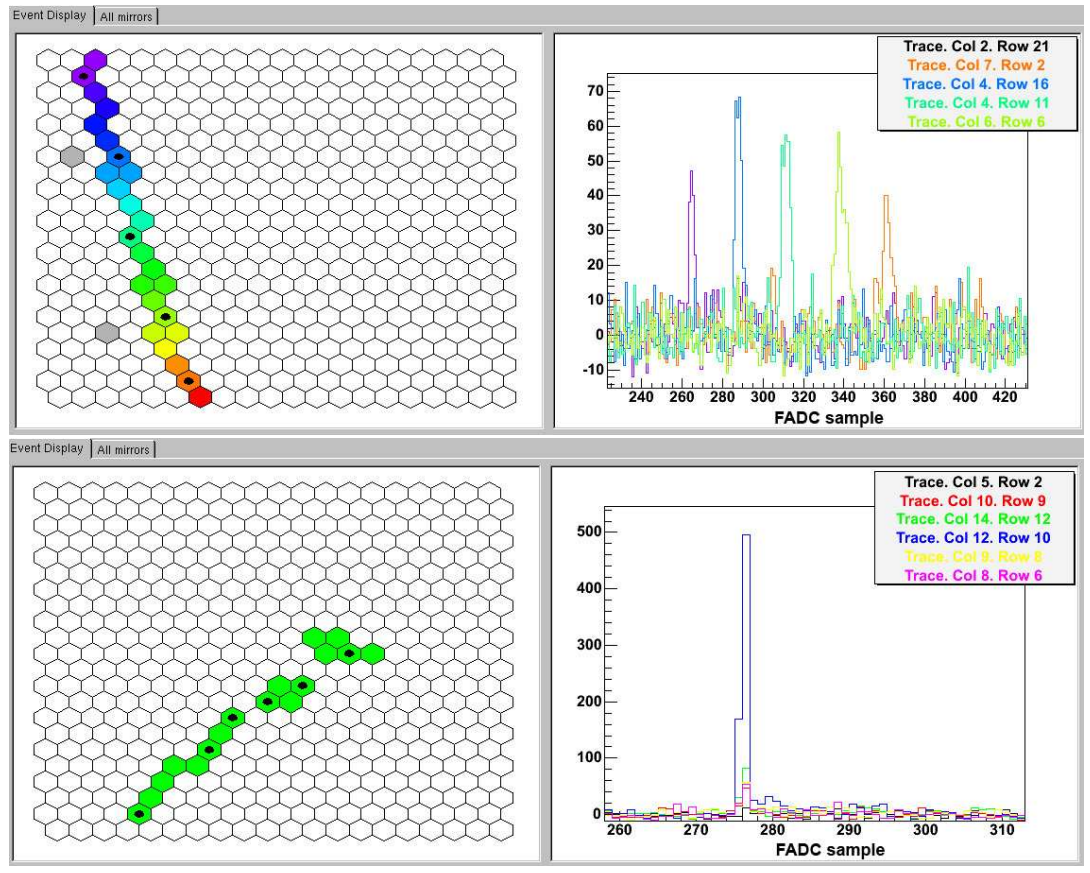

Figure 31: Top: A cosmic ray shower event as it appears in the event display. The pattern of the activated pixels is shown in the left panel while the right panel exhibits the response of the selected pixels as a function of time for the pixels marked by a black dot. The bin size is 100 ns. The development of the shower in the atmosphere can be qualitatively seen. Bottom: A background event most likely due to cosmic ray muon interacting with the glass of the PMT. All activated pixels give signals at the same time, a feature which is not compatible with a cosmic ray shower.

the Central Laser Facility (CLF), described in Ref. [9], the SDP reconstruction error can be evaluated by comparing the space angle between the normal vector to the experimentally determined SDP and the known true normal vector. This uncertainty in the SDP is of the order of a few tenths of a degree depending on, for example, the length of the observed track in the camera.

Next, the timing information of the pixels is used for reconstructing the shower axis within the SDP. As illustrated in Fig. 33, the shower axis can be characterized by two parameters: the perpendicular distance $R_{p}$ from the camera to the track, and the angle $\chi_{0}$ that the track makes with the horizontal line in the SDP. Each pixel which observes the track has a pointing direction which makes an angle $\chi_{i}$ with the horizontal line. Let $t_{0}$ be the time when the shower front on the axis passes the point of closest approach $R_{p}$ to the camera. The light arrives at the $i^{\text {th }}$ pixel at the time

$$
t_{i}=t_{0}+\frac{R_{p}}{c} \tan \left[\left(\chi_{0}-\chi_{i}\right) / 2\right]
$$




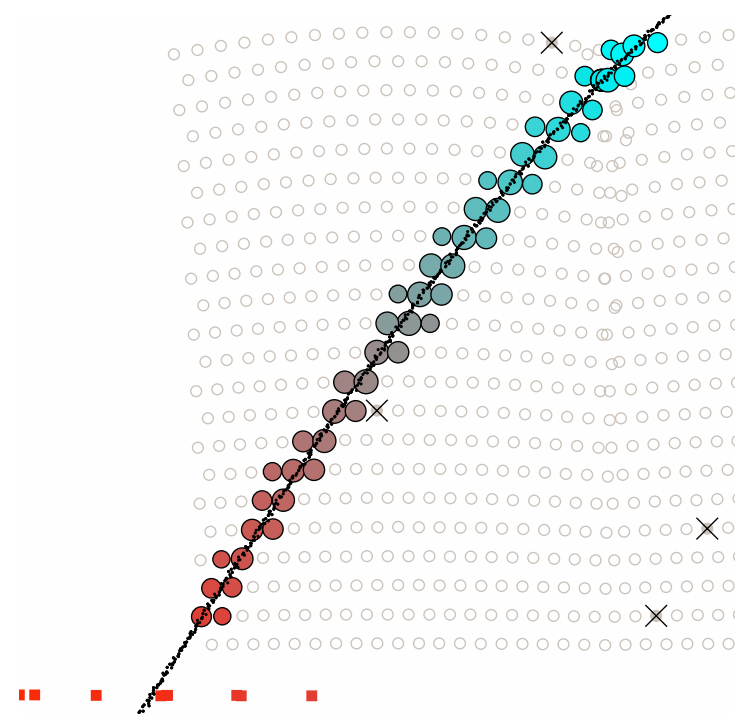

Figure 32: Light track of a hybrid event as seen by the fluorescence telescopes. The different colors indicate the timing sequence of the triggered pixels. The full line is the fitted showerdetector plane. (See text for explanation.) The red squares in the bottom left represent the surface stations that also triggered in this event. The crosses mark camera pixels that had a signal within the time of the trigger, but were marked by the reconstruction algorithm as too far either in distance (to the shower-detector plane) or in time (to the time fit).

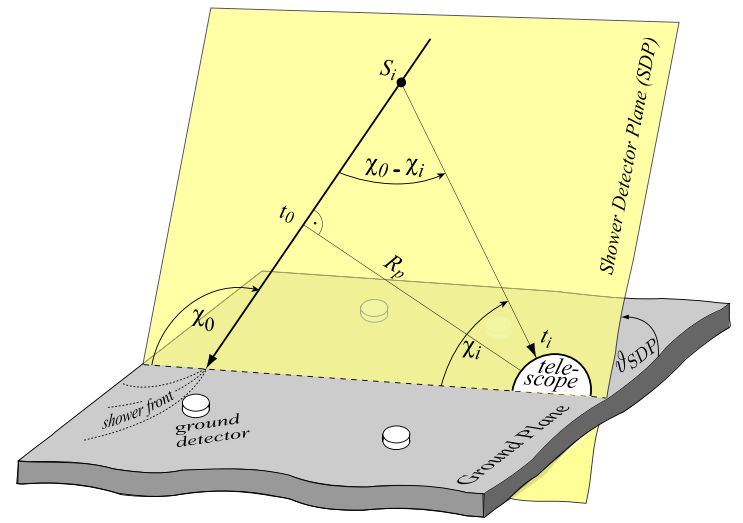

Figure 33: Illustration of the geometrical shower reconstruction from the observables of the fluorescence detector [43].

The shower parameters are determined by fitting the data points to this functional form. Using the fast sampling electronics, such a monocular reconstruction may achieve excellent accuracy. However, the accuracy of the monocular reconstruction is limited when the measured angular speed $d \chi / d t$ 
does not change much over the observed track length. An example is shown in Fig. 34. For these events (usually short tracks) there is a small curvature in the functional form of Eq. (11) such that there is a family of possible $\left(R_{p}, \chi_{0}\right)$ axis solutions. $R_{p}$ and $\chi_{0}$ are tightly correlated, but neither value is well constrained. This leads to uncertainty in other shower parameters, including the reconstructed shower energy.

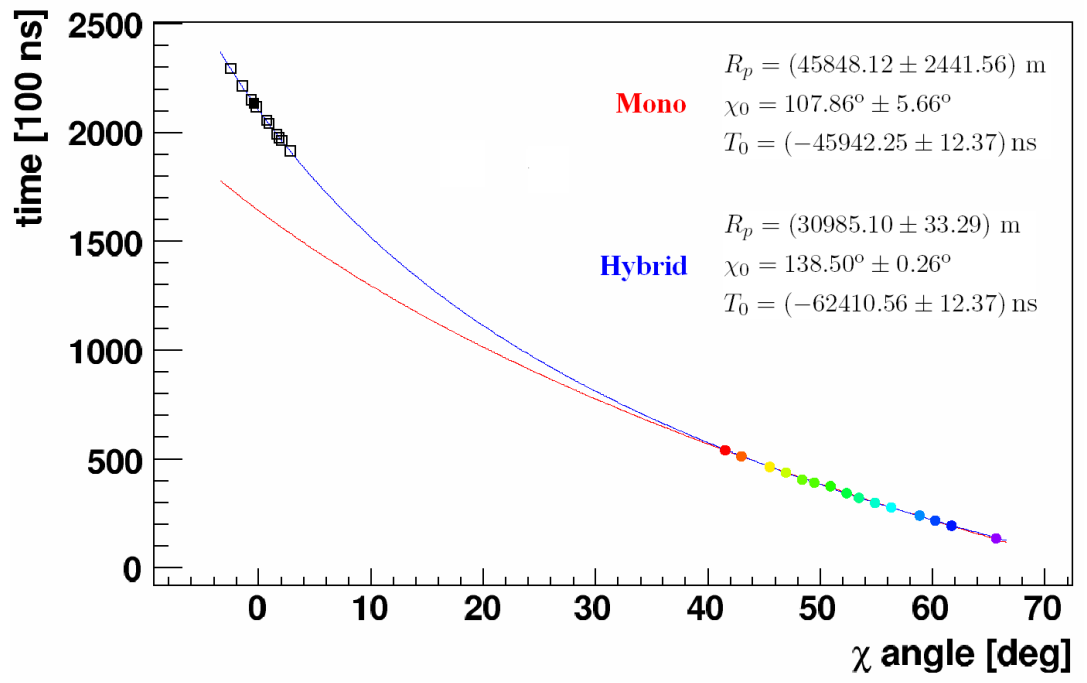

Figure 34: Functional form that correlates the time of arrival of the light at each pixel with the angle between the pointing direction of that particular pixel and the horizontal line within the shower-detector plane. FD data (color points) and SD data (squares) are superimposed to the monocular (red line) and hybrid (blue line) reconstruction fits. The full square indicates the SD station with the highest signal. This is a typical event in which the monocular reconstruction does not work well.

The fit degeneracy can be broken by combining the timing information from the SD stations with that of the FD telescopes. This is called the hybrid reconstruction. The hybrid solution for the example shown in Fig. 34 is shown as a blue line and the uncertainties in the parameters are specified in the legend.

Since the SD operates with a $100 \%$ duty cycle, most of the events observed by the FD are in fact hybrid events. There are also cases where the fluorescence detector, having a lower energy threshold, promotes a sub-threshold array trigger (see section 4.2.2). Surface stations are then matched by timing and location. This is an important capability because these sub-threshold hybrid events would not have triggered the array otherwise. In fact, the time of arrival at a single station at ground can suffice for the hybrid reconstruction.

The reconstruction uncertainties are validated using events with known geometries, i.e. light scattered from laser pulses. Since the location of the CLF (approximately equidistant from the first three fluorescence sites) and the direction of the laser beam are known to an accuracy better than the expected 
angular resolution of the fluorescence detector, laser shots from the CLF can be used to measure the accuracy of the geometrical reconstruction. Furthermore, the laser beam is split and part of the laser light is sent through an optical fiber to a nearby surface array station. Thus, the axis of the laser light can be reconstructed both in monocular mode and in the single-tank hybrid mode.

Using the timing information from the telescope pixels together with the surface stations to reconstruct real air showers, a core location resolution of $50 \mathrm{~m}$ is achieved. The resolution for the arrival direction of cosmic rays is $0.6^{\circ}$ [42]. These results for the hybrid accuracy are in good agreement with estimations using analytic arguments [44], measurements on real data using a bootstrap method [45], and previous simulation studies [46].

\subsection{Shower Profile and Energy Reconstruction}

Once the geometry of the shower is known, the light collected at the aperture as a function of time can be converted to energy deposit at the shower as a function of slant depth. For this purpose, the light attenuation from the shower to the telescope needs to be estimated and all contributing light sources need to be disentangled 47]: fluorescence light [48, 49, 50, 51], direct and scattered Cherenkov light [52, 53] as well as multiple-scattered light [54, 55].

An example of the measured light at the telescope aperture and the reconstructed light contributions and energy deposit profile is shown in Figs. 35. and 36 .

The calorimetric energy of a shower is estimated by fitting a Gaisser-Hillas function [56] to the reconstructed energy deposit profile and integrating it. Finally, the total energy of the shower is obtained by correcting for the 'invisible energy' carried away by neutrinos and high energy muons [57]. After quality selection, the energy resolution (defined as event-to-event statistical uncertainty) of the fluorescence detector is $\leq 10 \%$ [58].

\subsection{Detector Exposure}

The detection volume of fluorescence detectors varies with energy, as showers with higher energies emit more light and can be detected further away from the detector. The aperture also depends on environmental factors such as night-sky background light and atmospheric conditions. To model these effects, large sets of detailed MC simulations are used. The response of the Auger fluorescence telescopes has been simulated and the detector aperture has been estimated as a function of energy, average atmospheric conditions, and primary cosmic ray mass for a fixed configuration: a fully built detector with four fluorescence detectors and a $3000 \mathrm{~km}^{2}$ surface array [39, 40]. The aperture increases from approximately $900 \mathrm{~km}^{2}$ sr at $10^{17.5} \mathrm{eV}$ to about $7400 \mathrm{~km}^{2}$ sr at $10^{19} \mathrm{eV}$.

However, the more important quantity here is the hybrid detector exposure which accounts for both the time variability of the atmospheric conditions and the detector setup. Very detailed time-dependent detector Monte Carlo simulations are used to reproduce the actual data taking conditions of all components of the Pierre Auger Observatory and to derive the hybrid exposure. To assure 


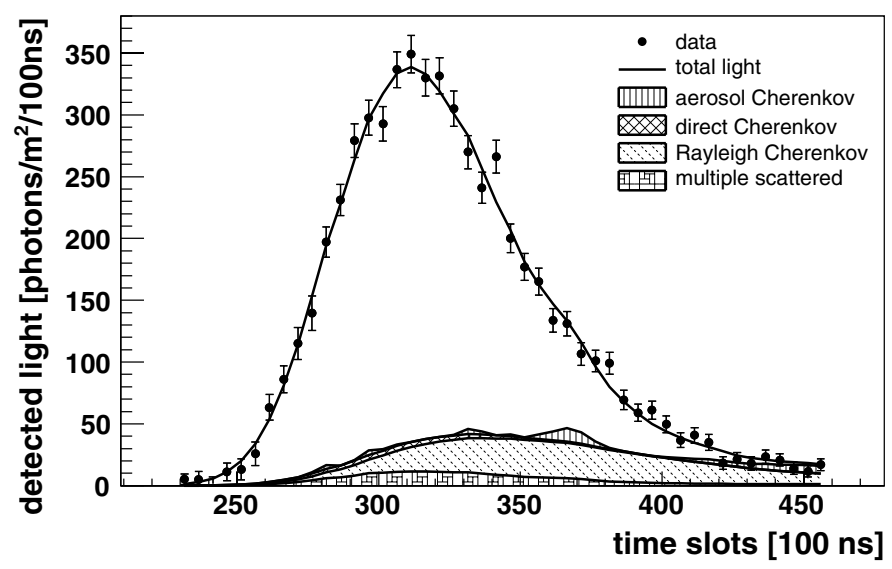

Figure 35: Example of a light-at-aperture measurement (dots) and reconstructed light sources (hatched areas).

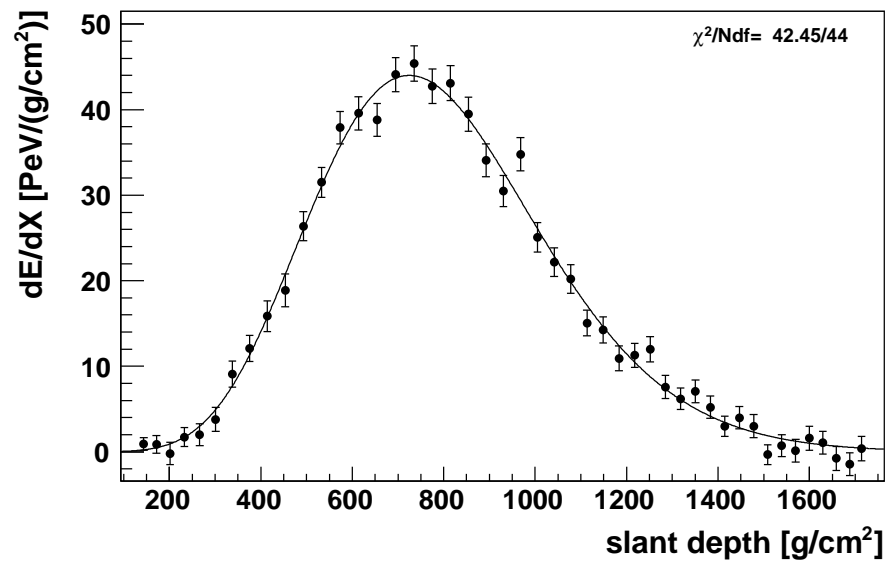

Figure 36: Energy deposit profile reconstructed from the light at aperture shown in Fig. 35 The line shows a Gaisser-Hillas fit of the profile. The energy reconstruction for this shower was $3.0 \pm 0.2 \cdot 10^{19} \mathrm{eV}$.

good agreement between data and Monte Carlo, extensive comparisons are performed at all reconstruction levels.

To limit the influence of trigger threshold effects, an energy-dependent fiducial volume has been defined and strict quality criteria are applied during the determination of the exposure 41]. For example, only data with a successful hybrid geometry reconstruction are selected, and the SD station used for the reconstruction has to lie within $750 \mathrm{~m}$ of the shower axis. This condition ensures 
that the probability to trigger at least one surface station is almost equal to one, and it significantly reduces the influence of the a-priori unknown primary mass composition. The resulting hybrid exposure, after all selection cuts accumulated during the 3 years of the building phase of the observatory, increases from about $50 \mathrm{~km}^{2}$ sr yr at $10^{18} \mathrm{eV}$ to about $550 \mathrm{~km}^{2}$ sr yr at $10^{19.5} \mathrm{eV}$.

\section{Summary}

The Observatory has been in scientific operation since late 2003, and the synergy between the surface array and the fluorescence detector has proved to be most fruitful. In particular, the calorimetric FD energy measurements have provided a calibration for the high-statistics data set obtained with full-time operation of the surface array.

This paper has described the Auger fluorescence detector system - its hardware, performance, calibration, and event reconstruction methods. The 24 wide-field telescopes view the atmosphere above the entire $3000-\mathrm{km}^{2}$ surface array. The Auger Observatory has opened a new chapter in cosmic ray physics because of both the large number of recorded high energy air showers and the quality of the hybrid measurements. The growing data set is expected to resolve the important questions relating to the highest energy particles of the universe.

\section{Acknowledgments}

The successful installation and commissioning of the Pierre Auger Observatory would not have been possible without the strong commitment and effort from the technical and administrative staff in Malargüe.

We are very grateful to the following agencies and organizations for financial support: Comisión Nacional de Energía Atómica, Fundación Antorchas, Gobierno De La Provincia de Mendoza, Municipalidad de Malargüe, NDM Holdings and Valle Las Leñas, in gratitude for their continuing cooperation over land access, Argentina; the Australian Research Council; Conselho Nacional de Desenvolvimento Científico e Tecnológico (CNPq), Financiadora de Estudos e Projetos (FINEP), Fundação de Amparo à Pesquisa do Estado de Rio de Janeiro (FAPERJ), Fundação de Amparo à Pesquisa do Estado de São Paulo (FAPESP), Ministério de Ciência e Tecnologia (MCT), Brazil; AVCR AV0Z10100502 and AV0Z10100522, GAAV KJB300100801 and KJB100100904, GACR 202/06/P006, MSMT-CR LA08016, LC527, 1M06002 and MSM0021620859, Czech Republic; Centre de Calcul IN2P3/CNRS, Centre National de la Recherche Scientifique (CNRS), Conseil Régional Ile-de-France, Département Physique Nucléaire et Corpusculaire (PNC-IN2P3/CNRS), Département Sciences de l'Univers (SDU-INSU/CNRS), France; Bundesministerium für Bildung und Forschung (BMBF), Deutsche Forschungsgemeinschaft (DFG), Finanzministerium Baden-Württemberg, Helmholtz-Gemeinschaft Deutscher Forschungs- zentren (HGF), Ministerium für Wissenschaft und Forschung, Nordrhein-Westfalen, Ministerium für Wissenschaft, Forschung und Kunst, 
Baden-Württemberg, Germany; Istituto Nazionale di Fisica Nucleare (INFN), Ministero dell'Istruzione, dell'Università e della Ricerca (MIUR), Italy; Consejo Nacional de Ciencia y Tecnología (CONACYT), Mexico; Ministerie van Onderwijs, Cultuur en Wetenschap, Nederlandse Organisatie voor Wetenschappelijk Onderzoek (NWO), Stichting voor Fundamenteel Onderzoek der Materie (FOM), Netherlands; Ministry of Science and Higher Education, Grant Nos. 1 P03 D 014 30, N202 090 31/0623, and PAP/218/2006, Poland; Fundação para a Ciência e a Tecnologia, Portugal; Ministry for Higher Education, Science, and Technology, Slovenian Research Agency, Slovenia; Comunidad de Madrid, Consejería de Educación de la Comunidad de Castilla La Mancha, FEDER funds, Ministerio de Ciencia e Innovación, Xunta de Galicia, Spain; Science and Technology Facilities Council, United Kingdom; Department of Energy, Contract No. DE-AC02-07CH11359, National Science Foundation, Grant No. 0450696, The Grainger Foundation USA; ALFA-EC / HELEN, European Union 6th Framework Program, Grant No. MEIF-CT-2005-025057, European Union 7th Framework Program, Grant No. PIEF-GA-2008-220240 and UNESCO.

\section{References}

[1] A. A. Watson, Proc. 30th ICRC (2007), arXiv:0801.2321 [astro-ph].

[2] I. Allekotte et al. [Pierre Auger Collaboration], Nucl. Instrum. Meth. A 586 (2008) 409.

[3] J. Abraham et al. [Pierre Auger Collaboration], submitted to Astropart. Phys (2009).

[4] R.M. Baltrusaitus et al., Nucl. Instrum. Meth. Phys. Res. A 240 (1985) 410.

[5] T. Abu-Zayyad et al., Nucl Instrum. Meth. Phys. Res. A 450 (2000) 253.

[6] H. Kawai et al., Nucl. Phys. Proc. Suppl. 175-176 (2008) 221.

[7] M. Nagano et al., Astropart. Phys. 22, 235 (2004).

[8] M. Ave et al., Astropart. Phys. 28, 41 (2007).

[9] B. Fick et al. [Pierre Auger Collaboration], JINST 1 (2006) P11003.

[10] Schott Glaswerke, Mainz, Germany (http://www.schott.com).

[11] D. Malacara, Optical shop testing, 3rd edition, Wiley-Interscience (2007)

[12] M. A. L. de Oliveira, V. de Souza, H. C. Reis and R. Sato, Nucl. Instrum. Meth. A 522 (2004) 360.

[13] R. Sato, C. O. Escobar [Pierre Auger Collaboration], Proc. 29th ICRC (2005), FERMILAB-CONF-05-285-AD-E-TD. 
[14] Schwantz Ferramentas Diamantadas e Comércio Óptico Ltda, Indaiatuba, Brazil.

[15] S. Agostinelli et al. [GEANT4 Collaboration], Nucl. Instrum. Meth. A 506 (2003) 250.

[16] M. G. Pia [Geant4 Collaboration], Nucl. Phys. Proc. Suppl. 125 (2003) 60.

[17] M. Ambrosio et al., Nucl. Instrum. Meth. A 478 (2002) 125.

[18] C. De Donato et al., Astroparticle Physics 28 (2007) 216.

[19] PHOTONIS, http://www.photonis.com.

[20] S. Argiro et al., Nucl. Instrum. Meth. A 461 (2001) 440.

[21] K. H. Becker et al., Nucl. Instrum. Meth. A 576 (2007) 301.

[22] Intratec GmbH, Beim Haferhof 5, D-25479 Ellerau; www.intratec.de.

[23] H. Gemmeke, A. Grindler, H. Keim, M. Kleifges, N. Kunka, D. Chernyakhovsky and Z. Szadkowski, IEEE Trans. Nucl. Sci. 47 (2000) 371.

[24] The FireWire interface is specified as IEEE 1394, see e.g. the book Don Anderson, 'FireWire System Architecture, Second Edition IEEE 1394a MindShare, Inc.', Addison Wesley, ISBN 0-201-48534-4.

[25] V. Scherini [Pierre Auger Collaboration], Proc. of the 20th European Cosmic Ray Symposium, Lisboa, Portugal (2006), ecrs-06-s0-187

[26] H. Gemmeke, M. Kleifges, A. Kopmann, N. Kunka, A. Menshikov, and D. Tcherniakhovski, Proc. 27th ICRC (2001), p737.

[27] M. Kleifges et al., IEEE TNS Vol. 50 No 4 (2003) P1204-1207.

[28] Z. Szadkowski, Nucl. Instrum. Meth. A 465 (2001) 540.

[29] A. Schmidt et al., "Third Level Trigger for the Fluorescence Telescopes of the Pierre Auger Observatory", subm. for publication to Nucl. Instr. Meth. A.

[30] J. T. Brack, R. Meyhandan, G. J. Hofman and J. Matthews, Astropart. Phys. 20 (2004) 653.

[31] Nichia America Corp., NSHU550 UV LED.

[32] Luxeon V Star, Document DS30, available at www.lumileds.com.

[33] National Institute of Standards and Technology, U.S. Dept. of Commerce, Calibration Program, Gaithersburg, MD 20899-2330; NIST Special Publication 250-41, 1998. 
[34] M. D. Roberts [Auger Collaboration], Proc. 28th (ICRC 2003), arXiv:astro$\mathrm{ph} / 0308410$.

[35] R. Knapik et al., Proc. 30th ICRC (2007) arXiv:0708.1924 [astro-ph].

[36] LS-1130-4 1100 Series FlashPac with FX-1160 xenon flash-lamp with reflector and borosilicate window from Perkin Elmer Opto-electronics, 35 Congress St., Salem, MA 01970.

[37] A. Rovero et al., for the Pierre Auger Collaboration, Astropart. Phys. 31, 305 (2009).

[38] Julian Rautenberg et al. [Pierre Auger Collaboration], Proc. 30th ICRC (2007), Vol. 5, p. 993

[39] L. Prado et al., Nucl. Instrum. Meth. A 545 (2005) 632.

[40] J. A. Bellido et al. [Pierre Auger Collaboration], Proc. 29th ICRC (2005), arXiv:astro-ph/0507103.

[41] L. Perrone [Pierre Auger Collaboration], Proc. 30th ICRC (2007), arXiv:0706.2643 [astro-ph].

[42] C. Bonifazi [Pierre Auger Collaboration], Proc. 29th ICRC (2005), FERMILAB-CONF-05-301-E-TD.

[43] D. Kuempel, K. H. Kampert and M. Risse, Astropart. Phys. 30 (2008) 167 arXiv:0806.4523 [astro-ph].

[44] P. Sommers, Astropart. Phys. 3, 349 (1995).

[45] B. Fick [Pierre Auger Collaboration], Proc. 28th ICRC (2003), arXiv:astro$\mathrm{ph} / 0308512$.

[46] B. R. Dawson, H. Y. Dai, P. Sommers and S. Yoshida, Astropart. Phys. 5, 239 (1996).

[47] M. Unger, B. R. Dawson, R. Engel, F. Schussler and R. Ulrich, Nucl. Instrum. Meth. A 588 (2008) 433.

[48] M. Nagano, K. Kobayakawa, N. Sakaki and K. Ando, Astropart. Phys. 22 (2004) 235.

[49] M. Ave et al. [AIRFLY Collaboration], Nucl. Instrum. Meth. A 597 (2008) 50.

[50] M. Ave et al. [AIRFLY Collaboration], Nucl. Instrum. Meth. A 597 (2008) 46.

[51] D .Gora et al., Astropart. Phys. 24 (2006) 484. 
[52] M. Giller, G. Wieczorek, A. Kacperczyk, H. Stojek and W. Tkaczyk, J. Phys. G 30 (2004) 97.

[53] F. Nerling, J. Bluemer, R. Engel and M. Risse, Astropart. Phys. 24 (2006) 421 [arXiv:astro-ph/0506729].

[54] M. D. Roberts, J. Phys. G 31 (2005) 1291.

[55] J. Pekala et al., Nucl. Instr. Meth. A, in print (2009), arXiv:0904.3230 [astro-ph]

[56] T. K. Gaisser, A. M. Hillas, Proc. 15th ICRC (1977).

[57] H. M. J. Barbosa, F. Catalani, J. A. Chinellato and C. Dobrigkeit, Astropart. Phys. 22 (2004) 159.

[58] B. R. Dawson [Pierre Auger Collaboration], Proc. 30th ICRC (2007), arXiv:0706.1105 [astro-ph]. 